\title{
Critical manifold of the kagome-lattice Potts model
}

\author{
Jesper Lykke Jacobsen ${ }^{1,2}$ and Christian R. Scullard ${ }^{3}$ \\ ${ }^{1}$ LPTENS, École Normale Supérieure, 24 rue Lhomond, 75231 Paris, France \\ ${ }^{2}$ Université Pierre et Marie Curie, 4 place Jussieu, 75252 Paris, France \\ ${ }^{3}$ Lawrence Livermore National Laboratory, Livermore CA 94550, USA \\ E-mail: jesper.jacobsen@ens.fr, scullard1@llnl.gov
}

\begin{abstract}
.
Any two-dimensional infinite regular lattice $G$ can be produced by tiling the plane with a finite subgraph $B \subseteq G$; we call $B$ a basis of $G$. We introduce a two-parameter graph polynomial $P_{B}(q, v)$ that depends on $B$ and its embedding in $G$. The algebraic curve $P_{B}(q, v)=0$ is shown to provide an approximation to the critical manifold of the $q$-state Potts model, with coupling $v=\mathrm{e}^{K}-1$, defined on $G$. This curve predicts the phase diagram not only in the physical ferromagnetic regime $(v>0)$, but also in the antiferromagnetic $(v<0)$ region, where analytical results are often difficult to obtain. For larger bases $B$ the approximations become increasingly accurate, and we conjecture that $P_{B}(q, v)=0$ provides the exact critical manifold in the limit of infinite $B$. Furthermore, for some lattices $G$-or for the Ising model $(q=2)$ on any $G$-the polynomial $P_{B}(q, v)$ factorises for any choice of $B$ : the zero set of the recurrent factor then provides the exact critical manifold. In this sense, the computation of $P_{B}(q, v)$ can be used to detect exact solvability of the Potts model on $G$.

We illustrate the method for two choices of $G$ : the square lattice, where the Potts model has been exactly solved, and the kagome lattice, where it has not. For the square lattice we correctly reproduce the known phase diagram, including the antiferromagnetic transition and the singularities in the Berker-Kadanoff phase at certain Beraha numbers. For the kagome lattice, taking the smallest basis with six edges we recover a well-known (but now refuted) conjecture of F.Y. Wu. Larger bases provide successive improvements on this formula, giving a natural extension of Wu's approach. We perform large-scale numerical computations for comparison and find excellent agreement with the polynomial predictions. For $v>0$ the accuracy of the predicted critical coupling $v_{\mathrm{c}}$ is of the order $10^{-4}$ or $10^{-5}$ for the 6 -edge basis, and improves to $10^{-6}$ or $10^{-7}$ for the largest basis studied (with 36 edges).
\end{abstract}

We dedicate this article to Professor Fa-Yueh Wu on the occasion of his 80th birthday.

\section{Introduction}

The two-dimensional $q$-state Potts model [1] has been extensively investigated over the last sixty years [2] and has served as a testbed for the powerful tools of quantum integrability [3]. It is defined by the reduced hamiltonian

$$
-\beta \mathcal{H}=K \sum_{(i j) \in E} \delta\left(\sigma_{i}, \sigma_{j}\right),
$$


where $K$ is the dimensionless coupling between the spins $\sigma_{i}=1,2, \ldots, q$ that are defined on the the vertices $i \in V$ of some given connected graph $G=(V, E)$ with vertex set $V$ and edge set $E$. Here $\beta=1 / k_{\mathrm{B}} T$, where $T$ is the temperature and $k_{\mathrm{B}}$ the Boltzmann constant, and $\delta\left(\sigma_{i}, \sigma_{j}\right)$ is Kronecker's delta function. From now on we suppose that $G$ is a piece of a regular lattice, and we are primarily interested in the limit of an infinite two-dimensional lattice.

The partition function $Z$ corresponding to (1) is most conveniently expressed in the Fortuin-Kasteleyn representation [4]

$$
Z=\sum_{\sigma} \exp (-\beta \mathcal{H})=\sum_{A \subseteq E} v^{|A|} q^{k(A)},
$$

where $|A|$ denotes the number of edges in the subset $A$, and $k(A)$ is the number of connected components in the induced graph $G_{A}=(V, A)$. The temperature parameter $v=\mathrm{e}^{K}-1$ will be used instead of $K$ in the following. Note that the representation (2) makes sense for any $q \in \mathbb{R}$, although (11) initially supposed that $q \in \mathbb{N}$. We shall also admit any value of $v \in \mathbb{R}$, although only $v \geq-1$ corresponds to a real coupling, $K \in \mathbb{R}$.

On a given lattice $G$, the Potts model can in general only be exactly solved along certain curves in the $(q, v)$ plane. This is in sharp contrast with the Ising model (alias the $q=2$ state Potts model), which can be solved at any temperature $v$ [5]. Another major difference is that the Potts model has been solved only on a very few lattices, including the square [6] and triangular lattices [7] and certain decorated versions thereof [8]. On the contrary, the Ising model can be solved on essentially any lattice (provided that suitable boundary conditions, usually doubly periodic, are imposed).

A first step in the solution of the Potts model on $G$ is to determine the critical manifold - or critical frontier - by which we understand the points in $(q, v)$ space at which the model stands at a phase transition It is a remarkable fact that, at least in the solvable cases [6, 10, 7], the loci of exact solvability coincide precisely with the critical manifold. It is equally remarkable that the critical manifolds on the square [6, 10, triangular [7] and hexagonal (the dual of the triangular) lattices turn out to be given by simple algebraic curves:

$$
\begin{aligned}
& \left(v^{2}-q\right)\left(v^{2}+4 v+q\right)=0, \quad \text { (square lattice) } \\
& v^{3}+3 v^{2}-q \quad=0, \quad \text { (triangular lattice) } \\
& v^{3}-3 q v-q^{2} \quad=0 . \quad \text { (hexagonal lattice) }
\end{aligned}
$$

It is an outstanding question of lattice statistics to determine whether these features hold true more generally.

The case of the Potts model on the kagome lattice has attracted particular attention. This is due not only to the practical applications of this model, but also to the fact that

$\ddagger$ The exact solution on the square lattice [6] shows that in the ferromagnetic regime $(v>0)$ the nature of the phase transition is first order for $q>4$, and second order (continuous) for $0 \leq q \leq 4$. The belief that this is true on any lattice is supported by other exact solutions [7] and general field theoretical arguments. The nature of the transitions for $q<0$ and/or $v<0$ is obviously a more delicate question, which has been addressed only in the simplest cases 9 . 
the kagome lattice is the "simplest" lattice on which an exact solution has not yet been found. More than thirty years ago Wu conjectured [11] that the critical manifold is given by the sixth-order algebraic curve

$$
v^{6}+6 v^{5}+9 v^{4}-2 q v^{3}-12 q v^{2}-6 q^{2} v-q^{3}=0 . \quad \text { (kagome lattice) }
$$

The derivation of (6) relied on a number of exact equivalences and - crucially - a certain non-rigorous homogeneity assumption. $\$$

The curve (66) has indeed a certain number of pleasing features:

(i) For $q=2$ it factorises as $(v+1)^{2}\left(v^{4}+4 v^{2}-8 v-8\right)=0$. This situates the ferromagnetic phase transition at $v_{\mathrm{c}}=\sqrt{3+2 \sqrt{3}}-1$, in agreement with the exact solution [13]

(ii) It passes through the origin $(q, v)=(0,0)$ with infinite slope, giving as expected a model of spanning trees (see [15]).

(iii) It behaves asymptotically as $v^{2} \propto q$ for $q \gg 1$, ensuring as expected first-order coexistence between the phases dominated by the terms $A=E$ and $A=\emptyset$ in (2).

Subsequent numerical results have however unambiguously established that (6) is not an exact result:

(i) For $q \rightarrow 1$ it places the bond percolation threshold at $p_{\mathrm{c}}=\frac{v_{\mathrm{c}}}{1+v_{\mathrm{c}}}=0.524429717 \cdots$, which is not correct but close enough that it took many years to be definitively ruled out [17]. Recent numerics [20] gives $p_{\mathrm{c}}=0.524404978(5)$.

(ii) For $q=3$ it gives $v_{\mathrm{c}}=1.876269208 \cdots$. This can be contrasted with the estimate [18] $v_{\mathrm{c}}=1.876456(40)$, obtained by analysing the low-temperature series for the magnetisation, susceptibility, zero-field partition function, and specific heat. Numerical diagonalisation of the transfer matrix provides the more accurate estimate [20] $v_{\mathrm{c}}=1.876458(3)$.

(iii) For $q=4$ the prediction of (6) is $v_{\mathrm{c}}=2.155842236 \cdots$. This cannot be discriminated by the less precise series result [18] $v_{\mathrm{c}}=2.1561(5)$, but is ruled out by the transfer matrix result [20] $v_{\mathrm{c}}=2.15620(5)$.

(iv) Discrepancies have also been observed for large $q \gg 4$ [19].

Below we shall present further conclusive numerical evidence against ([6). Despite this numerical refutation of the conjecture (6) approximation it is extraordinarily precise, the accuracy being of the order $10^{-4}$ or $10^{-5}$ (see also [20]).

$\S$ For completeness we mention that Tsallis [12] has proposed an alternative conjecture for the kagomelattice critical manifold. This has however been shown definitely not to be the exact expression. Moreover it is less accurate than Wu's conjecture (6).

\| The Ising model on the kagome and hexagonal lattices can be related by combining a star-triangle and a decoration-iteration transformation [14. It follows that $\left(1+v_{\text {kag }}\right)^{2}=2\left(1+v_{\text {hex }}\right)-1$ in our notations. Only one of the solutions to (5), $v_{\text {hex }}=1+\sqrt{3}$, leads to real solutions for $v_{\text {kag }}$, namely $v_{\text {kag }}=-1 \pm \sqrt{3+2 \sqrt{3}}$. 
In view of this evidence it is tempting to try to improve on (6) by systematically fitting the numerics to other low-order algebraic curves with reasonable (integer) coefficients. Such attempts [18, 21] have however proved inconclusive. Another line of research is to extend the approximation (6) to other (decorated) lattices of the kagome type [8, 20].

In this paper we present a general method for obtaining approximations to the critical manifold of the Potts models defined on any two-dimensional regular lattice $G$. The infinite lattice $G$ is obtained by tiling two-dimensional space by a certain finite subgraph $B \subseteq G$ that we shall refer to as the basis. The embedding of $B$ in $G$ determines exactly how $G$ is obtained as a tiling by the motif $B$ and will be defined precisely below. Corresponding to each embedded basis $B$ we define a two-parameter graph polynomial $P_{B}(q, v)$ which is closely related to the Tutte polynomial [23]; the approximation to the critical manifold then reads simply

$$
P_{B}(q, v)=0 .
$$

The precision of the approximation can be systematically improved by increasing the size, in a sense to be made more precise in section [7, of $B$.

The definition of $P_{B}(q, v)$ conceals a number of remarkable features:

(i) When not exact, the approximation (7) turns out to be very precise, even for the smallest possible choice of $B$. The analogue of (6) can thus be computed by hand rather easily for any regular lattice of interest.

(ii) For some lattices $G$ - or for the Ising model $(q=2)$ on any $G$-the polynomial $P_{B}(q, v)$ factorises for any choice of $B$. The zero set of the recurrent factor then turns out to provide the exact critical manifold. In this sense, the computation of $P_{B}(q, v)$ can be used to detect whether the Potts model on $G$ might be exactly solvable.

(iii) The determination of $P_{B}(q, v)$ for larger bases is well suited for exact computerassisted calculations. We shall pursue this point of view in section 3 below.

Here we illustrate the general method and the above remarkable features for the cases when $G$ is the square or the kagome lattice.

The square lattice serves as a benchmark, since its critical manifold is known completely [6, 10, 22, 9]. The result (3) is recovered by applying the general method to the simplest choice where the basis $B$ consists of 4 edges. We present extensions to larger bases consisting of 8, 16 and 32 edges that all contain the exact result (3) as a factor; the remaining factor gives information about the phase transitions at the Beraha numbers inside the antiferromagnetic regime [9].

In the case of the kagome lattice, the simplest case when $B$ consists of 6 edges reproduces Wu's conjecture (6) . We then present extensions to larger bases consisting of 12, 24 and 36 edges. These extensions systematically improve the agreement of the predicted critical manifold with the existing numerical results. In addition we present improved numerical results for the critical manifold-both in the ferromagnetic $(v>0)$ 
and antiferromagnetic $(v<0)$ regime - obtained by exact diagonalisation of the transfer matrix. Comparing this to the result from the 36-edge basis we find an accuracy of the order $10^{-6}$ or $10^{-7}$ in the ferromagnetic regime $(v>0)$, and both qualitative and quantitative improvements on (6) in the antiferromagnetic regime $(v<0)$.

The specialisation of these results to percolation (i.e., $\left.P_{B}(1, v)\right)$ has previously been reported by one of us 24]. Percolation polynomials for other lattices are reported in [25, 26, 27].

The paper is organised as follows. We begin the next section by reviewing the derivation of exact critical manifolds for three-terminal triangular-type lattices. We then show how these results may be used to define the graph polynomial $P_{B}(q, v)$ on any regular lattice by the contraction-deletion algorithm and use this to rederive Wu's conjectures [11] for the checkerboard and kagome critical manifolds. In section 3 we describe how this polynomial is calculated on larger bases using a computer program, and use the following sections to report polynomials computed for the square and kagome lattices on bases of up to 36 edges. In section [6 we support our conjecture that the polynomial approximations converge to the exact values by comparing our results with numerical calculations of critical curves.

\section{The graph polynomial}

The triangular-lattice Potts model with arbitrary interactions in up-pointing triangles is exactly solvable [29]. In particular, there exists a nice duality argument determining the critical manifold exactly. Since this is the starting point of our construction we begin by reviewing it.

\subsection{Triangular-lattice Potts model with interactions in up-pointing triangles}

Consider a Potts model on the triangular lattice in which all interactions occur inside up-pointing triangles, as shown in Fig. 19. Let the three spins around an up-pointing triangle $\Delta$ be labelled in cyclic order as $\sigma_{1}, \sigma_{2}, \sigma_{3}$, starting from the spin in the lowerleft corner (Fig. 1 b). The most general form of the local Boltzmann weight on $\Delta_{123}$, compatible with the $S_{Q}$ permutational symmetry of the spins, takes the form

$$
w_{123}=c_{0}+c_{1} \delta_{23}+c_{2} \delta_{13}+c_{3} \delta_{12}+c_{4} \delta_{123},
$$

where we have introduced the short-hand notation $\delta_{i j}=\delta\left(\sigma_{i}, \sigma_{j}\right)$ and $\delta_{i j k}=$ $\delta\left(\sigma_{i}, \sigma_{j}\right) \delta\left(\sigma_{j}, \sigma_{k}\right)$ for the Kronecker delta functions.

Each term in the sum can be represented graphically as follows:
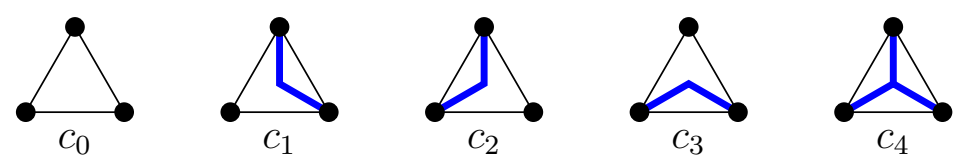

Expand now the product over $\Delta_{123}$ to form the partition function $Z$. This defines $Z$ in terms of clusters on the hexagonal lattice $G=(V, E)$ with local weights $c_{p}$. Each cluster 


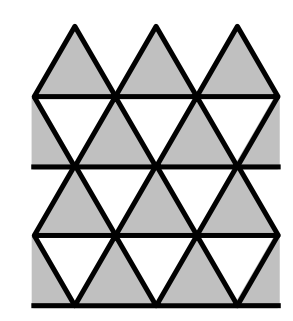

(a)

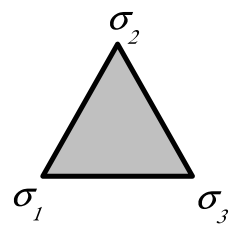

(b)

Figure 1. a) Triangular lattice with arbitrary interactions in up-pointing triangles. b) Labelling of spins around an up-pointing triangle $\Delta_{123}$.

comes with a weight $q$ from the summation over the spins $\sigma$, so that

$$
Z=\sum_{A \subseteq E} q^{k(A)} \prod_{p=0}^{4}\left(c_{p}\right)^{N_{p}},
$$

where $N_{p}$ is the number of up-triangles of type $c_{p}$, and $k(A)$ has the same meaning as in (2).

Alternatively, one can introduce loops that wrap around the boundaries and internal cycles of the clusters. The loops are represented graphically as follows:
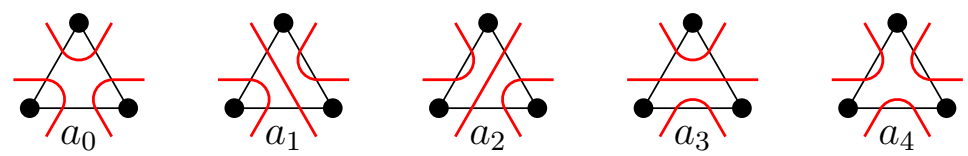

and a local weight $a_{p}$ is associated with each diagram. In down-pointing triangles, there is no interaction and each face is of the type $a_{0}$ (turned upside down). One has therefore a model of loops on a triangular lattice - shifted vertically by $1 / \sqrt{3}$ lattice spacings with respect to the one on which the Potts spins are defined - and each edge is covered by a loop.

Using the Euler relation the number of clusters $k(A)$ and the number of loops $l(A)$ are related by $2 k(A)=|V|-|A|+l(A)$, where we recall that $A$ is the set of blue edges on the hexagonal lattice $G=(V, E)$. The partition function in the loop representation therefore reads

$$
Z=n^{|V|} \sum_{\text {loops }} n^{l} \prod_{p=0}^{4}\left(a_{p}\right)^{N_{p}},
$$

where $n=\sqrt{q}$ is the weight of a loop, and the local weights $a_{p}$ and $c_{p}$ are related by

$$
\begin{aligned}
& a_{0}=c_{0}, \\
& n a_{i}=c_{i} \quad \text { for } i=1,2,3, \\
& n^{2} a_{4}=c_{4} .
\end{aligned}
$$

Following [29] we now suppose that at criticality the model is invariant under a $\pi / 3$ rotation of the triangular lattice where the loops live. This implies that the critical 
manifold is given by $a_{4}=a_{0}$ or [29]

$$
c_{4}=q c_{0} \text {. }
$$

Alternatively, the same relation can be found by a duality argument. Indeed, if we first transform the triangular lattice into a hexagonal lattice under duality, and next perform a decimation transformation of one half of the spins, we recover the original triangular lattice. Requiring that the weights be invariant under the combined transformation again leads to (14).

\subsection{Bases with three terminals}

As an example, we apply (14) to a triangular lattice with pure two-spin interactions, as in (2). We consider the case of arbitrary inhomogeneous two-spin couplings $\left\{v_{1}, v_{2}, v_{3}\right\}$ within $\Delta_{123}$, so that

$$
\begin{aligned}
& c_{0}=1, \\
& c_{i}=v_{i} \quad \text { for } i=1,2,3, \\
& c_{4}=v_{1} v_{2} v_{3}+v_{1} v_{2}+v_{2} v_{3}+v_{3} v_{1} .
\end{aligned}
$$

The critical manifold is then given by (14) in the form (7), where we have associated with the three-edge basis $B=\Delta_{123}$ the graph polynomial

$$
P_{B}\left(q,\left\{v_{1}, v_{2}, v_{3}\right\}\right)=v_{1} v_{2} v_{3}+v_{1} v_{2}+v_{2} v_{3}+v_{3} v_{1}-q .
$$

In the homogeneous case this indeed reduces to (4).

We can also recover the special case of the square lattice by setting $v_{3}=0$ in (16). This formally corresponds to a two-edge basis (remove one edge from $B$ ), and in the homogeneous case one obtains the first factor in (3).

It should be noted that the result (14) also applies when extra spins are present inside the up-pointing triangles One then simply sums out those spins to get an effective interaction of the form (8). As an example of this consider the hexagonal lattice with arbitrary inhomogeneous two-spin couplings $\left\{v_{1}, v_{2}, v_{3}\right\}$. We have then

$$
\begin{aligned}
& c_{0}=v_{1}+v_{2}+v_{3}+q, \\
& c_{1}=v_{2} v_{3}, \\
& c_{2}=v_{3} v_{1}, \\
& c_{3}=v_{1} v_{2}, \\
& c_{4}=v_{1} v_{2} v_{3} .
\end{aligned}
$$

The critical manifold again results from (14) in the form (17), with the graph polynomial

$$
P_{B}\left(q,\left\{v_{1}, v_{2}, v_{3}\right\}\right)=v_{1} v_{2} v_{3}-q\left(v_{1}+v_{2}+v_{3}+q\right) .
$$

In the homogeneous case we recover (5).

I This has been used extensively in [8]. One can also include explicit multi-spin interactions, but we shall not consider this possibility here. 
Note that (16) and (18) are related by the duality transformation [2] $v_{i} v_{i}^{*}=q$, up to an unimportant global factor. More precisely

$$
P_{B^{\text {tri }}}\left(q,\left\{v_{1}^{*}, v_{2}^{*}, v_{3}^{*}\right\}\right)=-\frac{q}{v_{1} v_{2} v_{3}} P_{B^{\text {hex }}}\left(q,\left\{v_{1}, v_{2}, v_{3}\right\}\right) .
$$

\subsection{Square-lattice Potts model with checkerboard interactions}

Up to this point our determinations of the critical manifolds have been exact. We shall now see that crucial new ingredients appear when we consider larger bases. In particular we pay attention to the way the basis $B$ is embedded in $G$ so as to tile the entire lattice.

Let us call a vertex of $B$ a terminal if it will have to be identified with a vertex of one or more copies of $B$ in the tiling of $G$. The remaining vertices of $B$ are called internal. The models discussed in section 2.1 used a basis with 3 terminals, and in the embedding of $B$ as up-pointing triangles each terminal was glued to a terminal in two other copies of $B$. This identification of each terminal with the other two is a convenient way of characterising the embedding.

It should be clear from the examples (15) and (17) that the derivation of the weights $c_{0}$ and $c_{4}$ are closely reminiscent of the computation of the partition function (2) itself. Indeed, these weights are just conditional probabilities that the terminals are connected in a certain way (not connected for $c_{0}$, and fully connected for $c_{4}$ ) in the Fortuin-Kasteleyn expansion.

The partition function (2) for the Potts mode + defined on any graph $G=(V, E)$ can be computed by the contraction-deletion method (see [23]). Namely, let $e \in E$ be any edge in $G$. Denote by $G / e$ the graph obtained from $G$ by contracting $e$ to a point and identifying the vertices at its end points (if they are different); and denote by $G \backslash e$ the graph obtained from $G$ by deleting $e$. The terms in the sum (2) can be grouped in two disjoint classes, according to whether $e \in A$ or $e \notin A$. For the terms with $e \in A$ (resp. $e \notin A$ ), the number $k(A)$ is unchanged upon contracting (resp. deleting) $e$. So the obvious generalisation of (2) to the case of arbitrary edge-dependent weights satisfies

$$
Z_{G}(q,\{v\})=v_{e} Z_{G / e}(q,\{v\})+Z_{G \backslash e}(q,\{v\}) .
$$

Observing that $Z$ factorises over the components of a disconnected graph, and that $Z=q$ for an isolated vertex, the contraction-deletion formula (20) allows one to compute $Z_{G}(q,\{v\})$ recursively.

We now define the graph polynomial $P_{B}(q,\{v\})$ corresponding to bases $B$ with more than three terminals. It is computed recursively by applying the contraction-deletion formula (20) to decrease the size of the basis. In the process we can rearrange $B$ by identifying terminals from the embedding, and we shall avoid disconnecting $B$ (modulo the embedding). The initial condition is to replace any 3-terminal basis obtained in the recursion by its corresponding critical manifold, which has been obtained in section 2.2 .

+ In the mathematics literature, the Potts model partition function is known as the multivariate Tutte polynomial [23]. 


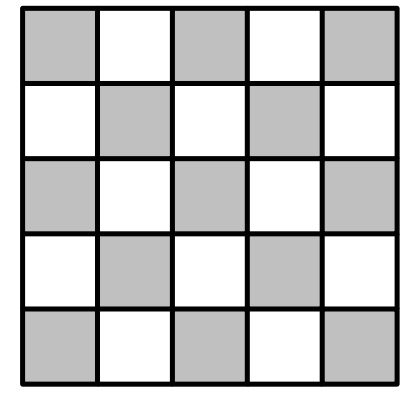

(a)

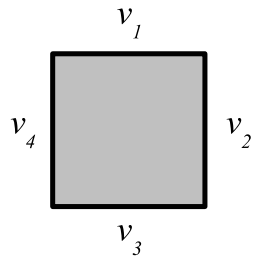

(b)

Figure 2. a) The square lattice with the checkerboard couplings shown in b).

Our key assumption - that we shall test in the following - is that $P_{B}(q,\{v\})=0$ provides an approximation to the critical manifold that becomes more and more precise (when it is not exact) as the size of $B$ increases.

Let us illustrate the above definition on a square-lattice Potts model with interactions in alternating faces forming a checkerboard pattern; see Fig. 2a. The corresponding basis has four terminals, and the embedding amounts to gluing diametrically opposite terminals. We are interested in the fully inhomogeneous case with couplings $\left\{v_{1}, v_{2}, v_{3}, v_{4}\right\}$, as shown in Fig. 2 $\mathrm{b}$. Performing the deletion-contraction of the edge with weight $v_{4}$ we obtain

$$
P_{B}\left(q,\left\{v_{1}, v_{2}, v_{3}, v_{4}\right\}\right)=v_{4} P_{B^{\operatorname{tri}}}\left(q,\left\{v_{1}, v_{2}, v_{3}\right\}\right)+P_{B_{\text {hex }}}\left(q,\left\{v_{1}, v_{2}, v_{3}\right\}\right) .
$$

In the first term the two terminals have been identified, so we arrive at the known case of the triangular lattice. In the second term we have made use of the embedding to flip one of the edges. In doing that, one of the terminals is turned into an internal vertex, and we recognise the 3-terminal basis of the hexagonal lattice. Substituting now (16) and (18) into (21) we find

$$
\begin{aligned}
P_{B}\left(q,\left\{v_{1}, v_{2}, v_{3}, v_{4}\right\}\right)= & v_{1} v_{2} v_{3} v_{4}+\left(v_{2} v_{3} v_{4}+v_{1} v_{3} v_{4}+v_{1} v_{2} v_{4}+v_{1} v_{2} v_{3}\right) \\
& -q\left(v_{1}+v_{2}+v_{3}+v_{4}\right)-q^{2}
\end{aligned}
$$

Note that this expression has an $S_{4}$ symmetry under the permutation of any two couplings.

The expression $P_{B}(q,\{v\})=0$ with (22) was first derived by Wu [1] using a different method (and a homogeneity assumption). He initially conjectured that it was the exact critical manifold. However, Enting [30] has observed that the putative $S_{4}$ symmetry is broken - albeit only at high order - in the series expansions for various physical quantities of the checkerboard-lattice Potts model, and he concluded that (22) cannot be correct 31]. In section 6.2 of [32] Maillard seems nevertheless to suggests that (22) may be correct. Unfortunately we do not know of any numerical investigation of the critical manifold for the completely inhomogenious checkerboard Potts model that would allow us to assess whether (22) is correct, and if not, how accurate an approximation it 
is. But in section 4 below we have computed the polynomials $P_{B}(q,\{v\})$ corresponding to larger bases for the square lattice, with four different couplings arranged in a checkerboard pattern. We observe that these polynomials invariably factorise, shedding the small factor (22). This is a strong indication that (22) is indeed the exact critical manifold of the checkerboard-lattice Potts model.

Something interesting happens if we specialise to the usual square lattice with horizontal couplings $v_{1}=v_{3}$ and vertical couplings $v_{2}=v_{4}$. Then (22) factorises as

$$
P_{B}\left(q,\left\{v_{1}, v_{2}, v_{1}, v_{2}\right\}\right)=\left(v_{1} v_{2}-q\right)\left(v_{1} v_{2}+2 v_{1}+2 v_{2}+q\right),
$$

and in the homogeneous case we recover (3). The zero set of (23) is known to be the exact critical manifold. Indeed, the first factor corresponds to the ferromagnetic transition curve [6] and the second one to the antiferromagnetic transition [10] (see also [22, 9]). It is particularly remarkable that the antiferromagnetic transition curve is obtained exactly by this approach, since - to our knowledge - it cannot be obtained by a simple duality argument. Indeed, [10] relies on the solution of the Yang-Baxter equation and a so-called $Z$-invariance.

\subsection{Wu's conjecture recovered}

We now turn to the kagome lattice $G$. The simplest choice of basis $B_{6}$ is the 4-terminal arrangement of six edges shown in Fig. $3 \mathrm{a}$ which produces $G$ by the checkerboard embedding discussed in section 2.3. Using the contraction-deletion identity (20) on the $v_{4}$ edge we obtain for the corresponding graph polynomial $K(\{v\})=P_{B_{6}}(q,\{v\})$ :

$$
\begin{aligned}
K\left(v_{1}, v_{2}, v_{3}, v_{4}, v_{5}, v_{6}\right)= & v_{4} B\left(v_{5}+v_{6}+v_{5} v_{6}, v_{1}, v_{2}, v_{3}\right) \\
& +A\left(v_{1}, v_{2}, v_{3}, v_{5}, v_{6}\right) .
\end{aligned}
$$

In the first term, the $v_{4}$ edge has been contracted, so that the $v_{5}$ and the $v_{6}$ edges are in parallel. Using parallel reduction [23] these can then be replaced by a single edge with coupling $v_{5}+v_{6}+v_{5} v_{6}$. The result is a 3 -terminal basis of the martini-B lattice [33, 34] (see Fig. 3b) whose graph polynomial we have denoted $B\left(v_{1}, v_{2}, v_{3}, v_{4}\right)$. This is then replaced by the critical manifold (14) which reads explicitly [35]

$$
\begin{aligned}
B\left(v_{1}, v_{2}, v_{3}, v_{4}\right)= & v_{1}\left(v_{2} v_{3}+v_{2} v_{4}+v_{3} v_{4}+v_{2} v_{3} v_{4}\right) \\
& -q\left(q+v_{1}+v_{3}+v_{4}\right) .
\end{aligned}
$$

In the second term in (24) the $v_{4}$ edge has been deleted. Flipping the $v_{5}$ and $v_{6}$ edges using the embedding, the result is a 3 -terminal basis of the martini-A lattice (see Fig. 3r) whose graph polynomial we have denoted $A\left(v_{1}, v_{2}, v_{3}, v_{4}, v_{5}\right)$. Using again (14) it is replaced by the critical manifold [35]

$$
\begin{aligned}
A\left(v_{1}, v_{2}, v_{3}, v_{4}, v_{5}\right)= & v_{4} v_{5}\left(v_{1} v_{2}+v_{2} v_{3}+v_{3} v_{1}+v_{1} v_{2} v_{3}\right) \\
& -q\left(q^{2}+q\left(v_{1}+v_{2}+v_{3}+v_{4}+v_{5}\right)+v_{1} v_{2} v_{3}\right. \\
& \left.+\left(v_{2}+v_{4}\right)\left(v_{3}+v_{5}\right)+v_{1}\left(v_{2}+v_{3}+v_{4}+v_{5}\right)\right) .
\end{aligned}
$$




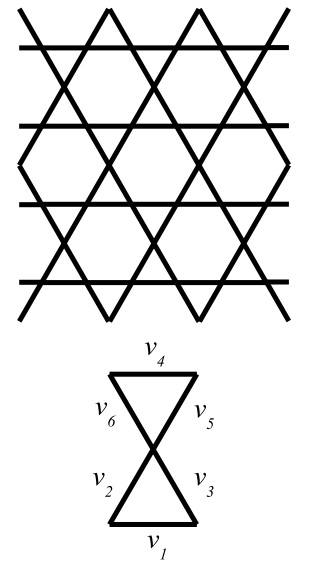

(a)
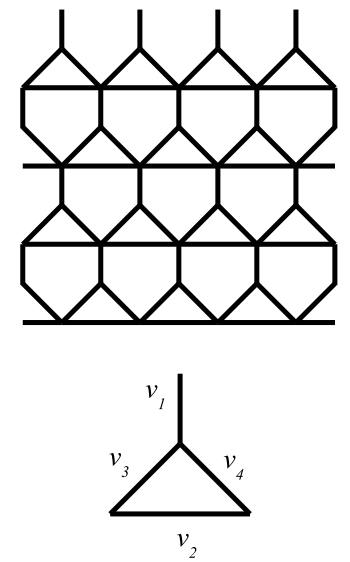

(b)
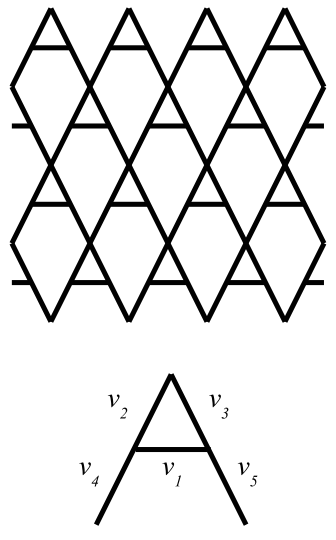

(c)

Figure 3. Assignment of interactions on a) the kagome lattice; b) the martini-B lattice; c) the martini-A lattice.

Inserting (25) and (26) into (24) produces the desired graph polynomial. The corresponding approximation for the critical manifold is $K(\{v\})=0$ by (17). The case of a kagome lattice with different couplings for each of the three principal directions corresponds to the choice $v_{4}=v_{1}, v_{5}=v_{2}$ and $v_{6}=v_{3}$. This coincides with Wu's most general conjecture for the critical manifold (see Eq. (11) in [11]) and reduces to (6) in the homogeneous case.

We have already discussed in the introduction that (6) is a very precise approximation to the critical manifold, but not an exact result. Further numerical evidence for this statement will be given in section 6 .

\section{General bases}

As remarked above, the general contraction-deletion formula for $P_{B}(q,\{v\})$ is identical to that for the partition function (see (20) ), namely

$$
P_{B}(q,\{v\})=v_{e} P_{B / e}(q,\{v\})+P_{B \backslash e}(q,\{v\}) .
$$

In the simple kagome example given above, both contraction and deletion yielded lattices for which the exact solution is known. For larger bases, this will not always be the case and then the algorithm must be applied to whichever (or both) of $B / e$ and $B \backslash e$ is unsolved, and so on recursively until solved lattices appear. This results in a sort of binary tree in which each node is a lattice and branches terminate on known graphs. However, the final polynomial is independent of the edge chosen at each step, and is therefore a unique property of the graph $B$ and the terminal identifications that define its embedding in $G$. Of course, the complexity of the problem increases exponentially with the number of edges in the basis, and thus the algorithm must be handled by computer for large bases. The operation of the program that implements the contraction-deletion algorithm can be sketched as follows: 
(i) As input, it takes an array of internal vertices, terminals and edges, along with the specification of their connectivities and identifications between terminals that determine how the basis is tiled to form the lattice.

(ii) An edge, $e$, is then chosen, respecting a limited set of criteria (e.g., not disconnecting the basis), for contraction and deletion to give the new bases $G / e$ and $G \backslash e$.

(iii) Often, these new graphs have edges doubled in series, or, as for the martini-B lattice in (24), in parallel. These may be simplified by replacing them by a single edge with an effective interaction. Other complications are also possible, such as isolated or dead-end vertices, and these are removed from the problem.

(iv) After simplifying, $G / e$ and $G \backslash e$ are checked against a small set of known lattices. These are the square, hexagonal and triangular lattices, along with the onedimensional chain and certain situations that are known to give critical manifolds that are identically zero (see [28]). We restrict ourselves to these lattices because of the relative ease of identification (e.g., all edges are equivalent and we are thus not required to worry about edge-matching). If a graph is recognised, it is replaced by the corresponding graph polynomial and the process ends. If not, step 2 is called recursively with the unknown graph as input.

(v) The final result is a tree of lattices with branches that terminate on known lattices. The output of the program is a list of functions giving the contractiondeletion formula for each lattice. This list is evaluated in MATHEMATicA and the homogeneous polynomial can then be found.

This program was used to compute percolation $(q=1)$ polynomials in [24] and [28]. Only minor adaptations were required to find the polynomials for general $q$, so we discuss only those changes here and refer the interested reader to [28] where the implementation is described in detail.

\subsection{Simplification}

The only differences between the percolation and Potts implementations are in the simplification phase of the algorithm, most importantly in the way interactions are relabelled upon the removal of doubled bonds. The handling of dead-end vertices also requires a minor modification.

3.1.1. Edges doubled in parallel. Contracting the $v_{1}$ edge in the martini-A lattice of Fig. 3r leaves $v_{2}$ and $v_{3}$ doubled in parallel. These must be replaced by a single edge. In the percolation computation, the probabilities $p_{2}$ and $p_{3}$ are combined into $1-\left(1-p_{2}\right)\left(1-p_{3}\right)=p_{2}+p_{3}-p_{2} p_{3}$, i.e., the probability that at least one bond is open. The Potts expression for the effective interaction, $v_{p}$, is similar [23]:

$$
v_{p}=v_{2}+v_{3}+v_{2} v_{3} .
$$


3.1.2. Edges doubled in series. For the martini-A lattice in Fig. 3k, deleting the $v_{1}$ edge leaves edges doubled in series, for example $v_{3}$ and $v_{5}$. These are replaced by the effective interaction, $v_{s}$, given by [23]:

$$
v_{s}=\frac{v_{3} v_{5}}{v_{3}+v_{5}+q} \text {. }
$$

The denominator in (29) presents a slight complication. Consider the deletion of $v_{1}$ in Fig. 3ic, giving the square lattice, which has the inhomogeneous manifold $S\left(\tilde{v}_{1}, \tilde{v}_{2}\right) \equiv$ $\tilde{v}_{1} \tilde{v}_{2}-q=0$, but with edges doubled in series. The contraction-deletion formula (27) works because the completely inhomogeneous polynomial is at most first-order in any interaction; we may have terms like $v_{1} v_{2} v_{3}$ but not $v_{1}^{2} v_{2}$ or, more to the point, $v_{1} /\left(v_{2}+v_{3}+q\right)$. As such, we cannot use

$$
S\left(\frac{v_{3} v_{5}}{v_{3}+v_{5}+q}, \frac{v_{2} v_{4}}{v_{2}+v_{4}+q}\right)
$$

for the manifold of $B \backslash e$. However, because the critical manifold is found by setting $S\left(\tilde{v}_{1}, \tilde{v}_{2}\right)=0$, we are free to multiply away these denominators, and we must do this before inserting an expression into the contraction-deletion formula. Thus, we have

$$
\begin{aligned}
& A\left(v_{1}, v_{2}, v_{3}, v_{4}, v_{5}\right)=v_{1} H\left(v_{2}+v_{3}+v_{2} v_{3}, v_{4}, v_{5}\right) \\
+ & \left(v_{3}+v_{5}+q\right)\left(v_{2}+v_{4}+q\right) S\left(\frac{v_{3} v_{5}}{v_{3}+v_{5}+q}, \frac{v_{2} v_{4}}{v_{2}+v_{4}+q}\right),
\end{aligned}
$$

from which we recover (26).

3.1.3. Dead-end vertices. An internal vertex connected to only one edge is a dead end. In percolation, such an edge contributes nothing to the connectivities between terminals, and therefore nothing to the graph polynomial. Its probability, $p$, disappears from the problem because it only appears in an overall factor of $[p+(1-p)]=1$ multiplying the probability of every event. So the edge is simply removed from the problem along with the vertex. In Potts language, the weight of every event is multiplied by $(v+q)$ and thus, although we still remove the edge and vertex, the manifold must be multiplied by this factor upon the removal. We give a simple example. In Fig. 4a , we have a triangular lattice with two edges doubled in series. Adhering to the rules set out in the previous section, the graph polynomial for this is given by

$$
\left(v_{3}+v_{4}+q\right) T\left(v_{1}, v_{2}, \frac{v_{3} v_{4}}{v_{3}+v_{4}+q}\right) .
$$

Contraction-deletion should also produce this answer. Contracting $v_{4}$ just gives the simple triangular lattice, $T\left(v_{1}, v_{2}, v_{3}\right)$, but deleting this edge results in the square lattice with a dead-end vertex and edge, $v_{3}$. We remove these from the problem but retain the factor of $\left(q+v_{3}\right)$ to give $\left(q+v_{3}\right) S\left(v_{1}, v_{2}\right)$. Injecting these into the contraction-deletion formula,

$$
v_{4} T\left(v_{1}, v_{2}, v_{3}\right)+\left(q+v_{3}\right) S\left(v_{1}, v_{2}\right),
$$

we do indeed recover (32), which can be seen by expanding both expressions. 


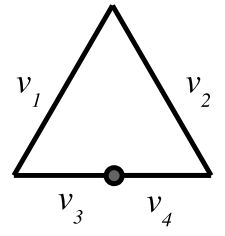

(a)

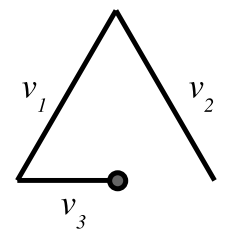

(b)

Figure 4. a) Triangular lattice with a series-doubled edge; b) deletion of the $v_{4}$ edge leaves $v_{3}$ and its right vertex as a dead end.

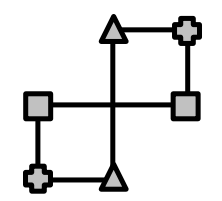

(a)

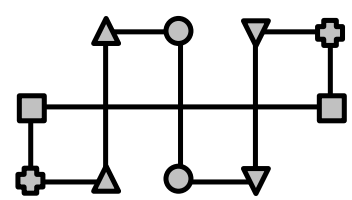

(b)

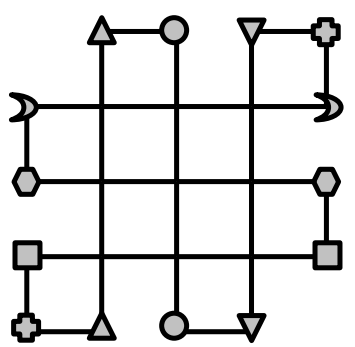

(c)

Figure 5. a) 8-edge; b) 16-edge; and c) 32-edge bases for the square lattice. Terminals with matching shapes are identified.

Aside from these changes, the main operation of the program, including edge selection and lattice identification, are exactly as described in [28]. We also have the same upper limit of practical feasibility, namely using basis with at most 36 edges.

\section{Square-lattice Potts model using larger bases}

Summarising, we have seen that the definition of $P_{B}(q, v)$ made in section 2.3 leads to known approximations of the critical manifold when the lattice $G$ is obtained from a 4terminal basis. In the remainder of the paper we shall corroborate our key assumption that by using larger bases the accuracy of the approximations can be improved, and more subtle features of the critical manifolds can be uncovered.

To this end we study first the benchmark example of the square lattice in this section. The following section will be devoted to the kagome lattice.

We first extend the 4-edge checkerboard to the 8-edge basis in Fig. 5a. The polynomial for this case is given by

$$
\left(q-v^{2}\right)\left(q+4 v+v^{2}\right)\left(q^{2}+4 q v+v^{4}+4 v^{3}+8 v^{2}\right),
$$

and we see the exact solutions appearing as factors. The polynomial for the 16-edge basis depicted in Fig. 55 is given in the Appendix in equation (A1). We also found the

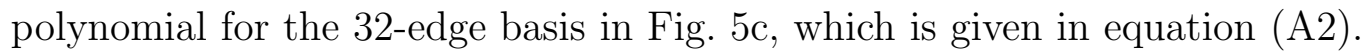




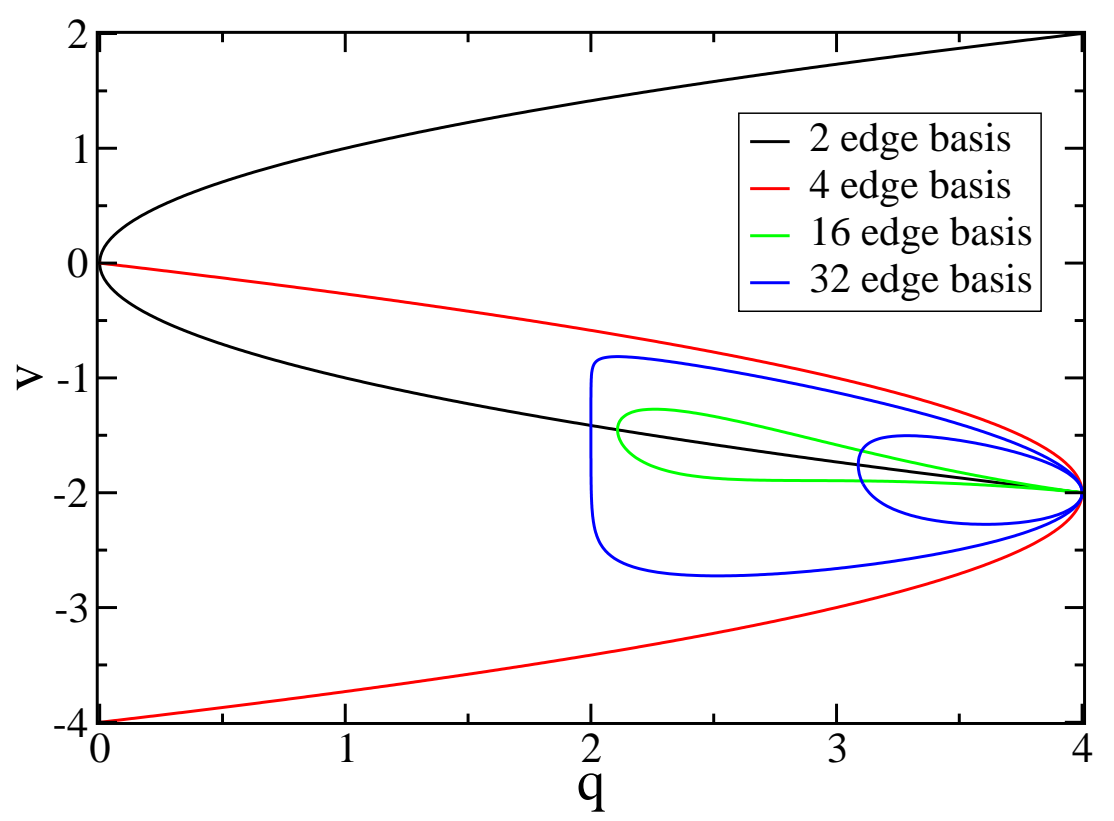

Figure 6. Manifolds $P_{B}(q, v)$ for various choices of bases $B$ for the square lattice (see text). The exact critical manifolds (3) obtained from the bases with 2 and 4 edges are contained in the manifolds corresponding to larger bases.

We notice for the polynomials (34), (A1) and (A2) that

$$
P_{B}(q, v)=-v^{|E|} q^{-|E| / 2} P_{B}\left(q, v^{*}\right)
$$

with $v^{*}=q / v$, where $|E|$ is the number of edges in the basis. This means that the graph polynomials respect the self-duality of the square lattice. In particular, the solutions to $P_{B}(q, v)=0$ are either self-dual curves, or pairs of mutually dual curves.

It appears that the exact solvability [6, 10, of the square-lattice Potts model manifests itself in the presence of the factors (3) in all the graph polynomials constructed from larger bases, irrespective of their embedding. More generally, we have found that for four different coupling constants arranged in a checkerboard pattern (see Fig. 2), the polynomials always contain the factor (22). It remains to assess whether the remaining (in general non-factorisable) factor in $P_{B}$ has any physical relevance.

To investigate this issue, we have plotted the manifolds $P_{B}(q, v)=0$ for the different bases in Fig. 6. It is seen that the remaining factor produces dual pairs of curves inside the dual pair of antiferromagnetic transition curves $v^{2}+4 v+q=0$, in the form of "bubbles" emanating from the point $(q, v)=(4,-2)$. Each bubble intersects the selfdual transition curve $v=-\sqrt{q}$ in two points, of which one is exactly $q_{0}=4$ and the other we denote $q_{\mathrm{c}}$. The bubbles are almost vertical in the vicinity of that latter point. For the 32-edge basis we find two bubbles with respectively

$$
\begin{aligned}
& q_{\mathrm{c}, 1}=2.000059024 \cdots, \\
& q_{\mathrm{c}, 2}=3.088542134 \cdots .
\end{aligned}
$$

These values are conspicuously close to 2 and 3 . 
It was shown in [22, 9] that the Potts model possesses singularities inside the socalled Berker-Kadanoff phase, which is the region bordered by the antiferromagnetic transition curves $v^{2}+4 v+q=0$. These singularities occur at the Beraha numbers $q=B_{k}$ with

$$
B_{k}=(2 \cos (\pi / k))^{2}, \quad \text { with } k=2,3,4, \ldots
$$

and are independent of $v$. For a given integer $k$ the singularity may or may not concern the dominant term in the partition function, and only the former case leads to a phase transition. The independence of $v$ implies that the incidence on the phase diagram is the formation of a vertical ray in the $(q, v)$ plane.

The issue of dominance depends on the choice of boundary conditions. In [36] it was argued from results of conformal field theory - and checked numerically - that with cyclic boundary conditions (free in one lattice direction and periodic in the other) vertical rays are formed for $k \in 2 \mathbb{N}$. The corresponding result for toroidal boundary conditions [37] (periodic in both lattice direction) is that vertical rays occur only for $k=4$ and $k=6$. Because of the identification of opposite terminals in the embeddings of the basis $B$ that we have used, it is the toroidal boundary conditions that are relevant in the present case.

In (36) ) we have indeed $q_{\mathrm{c}, 1} \approx B_{4}=2$ and $q_{\mathrm{c}, 2} \approx B_{6}=3$, in agreement with [37]. We therefore conjecture that in the limit of an infinitely large basis, $P_{B}(q, v)$ will contain (3) and a remaining factor whose zeros produce a couple of vertical rays at $q=2$ and

$q=3$, extending between the two branches of the antiferromagnetic transition curve $v^{2}+4 v+q=0$. The two bubbles obtained from the 32-edge basis provide strong support of this conjecture; we believe that the non-vertical parts of those bubbles will coincide with parts of the antiferromagnetic transition curve in the limit of infinite $B$.

To produce an almost vertical ray from $P_{B}(q, v)=0$ clearly requires the algebraic expression $P_{B}(q, v)$ to be of large degree in $q$ and $v$. Obviously the degree in $q$ equals the number of vertices in the basis $B$ (counted up to identification of terminals through the embedding), while the degree in $v$ equals the number of edges in $B$. The above conjecture is compatible with the increase of degree as the basis becomes larger: infinite-degree expressions are needed to produce truly vertical rays in the limit of an infinite basis.

\section{Kagome-lattice Potts model using larger bases}

We are now ready to fulfill the main objective of this paper, which is to improve on Wu's approximation (6) for the critical manifold of the Potts model on the kagome lattice. Starting with the 12-edge basis in Fig. $7 \mathrm{a}$, we find the polynomial

$$
\begin{aligned}
& \left(q^{3}+6 q^{2} v+12 q v^{2}+2 q v^{3}-9 v^{4}-6 v^{5}-v^{6}\right) \times \\
& \left(q^{3}+6 q^{2} v+16 q v^{2}+24 v^{3}+2 q v^{3}+17 v^{4}+6 v^{5}+v^{6}\right)=0 .
\end{aligned}
$$

The first term in brackets is once again Wu's formula (6), so, similar to the situation in percolation [24], we get no better estimate of the ferromagnetic critical point by extending from 6 to 12 edges. However, the second term does provide us with additional 


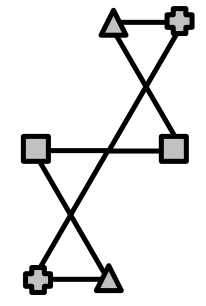

(a)

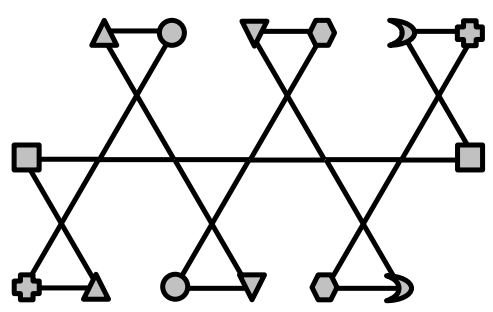

(c)

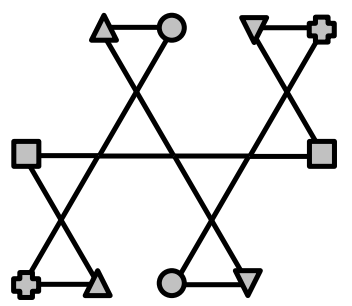

(b)

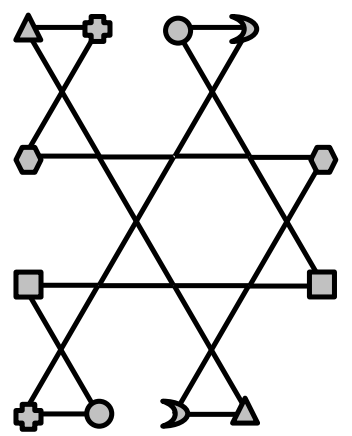

(d)

Figure 7. a) 12-edge; b) 24-edge; and c), d) 36-edge bases for the kagome lattice.

information in the antiferromagnetic region, which is plotted in red in Fig. 8. We also found the polynomials for the 24-edge basis of Fig. $7 \mathrm{~b}$, and the two different 36-edge bases of Fig. $7 \mathrm{c}$ and $7 \mathrm{~d}$. These polynomials are given in the Appendix (see eqs. (A3), (A4), and (A5)).

The manifolds $P_{B}(q, v)=0$ for the different bases are plotted in Fig. 8 .

In the ferromagnetic region $(v>0)$ the difference between the various curves is not visible on the scale of the figure. The approximations for the critical coupling in percolation read

$$
v_{\mathrm{c}}(q=1)= \begin{cases}1.102738621 \cdots & \text { (6-edge basis) } \\ 1.102636956 \cdots & \text { (24-edge basis) } \\ 1.102632538 \cdots & \text { (36-edge basis) }\end{cases}
$$

to be compared with the most precise numerical estimate [20] $v_{\mathrm{c}}(q=1)=$ $1.10262924(2)$. The corresponding percolation threshold is $p_{\mathrm{c}}=\frac{v_{\mathrm{c}}}{1+v_{\mathrm{c}}}$. For the Ising model $(q=2)$ all curves pass through the exact critical point [13] $v_{\mathrm{c}}=\sqrt{3+2 \sqrt{3}}-1=$ $1.542459756 \cdots$. When $q=3$ we have

$$
v_{\mathrm{c}}(q=3)= \begin{cases}1.876269208 \cdots & \text { (6-edge basis) } \\ 1.876439754 \cdots & \text { (24-edge basis) } \\ 1.876447147 \cdots & \text { (36-edge basis) }\end{cases}
$$

to be compared with the numerical estimate [20] $v_{\mathrm{c}}(q=3)=1.876458(3)$. Finally, for 


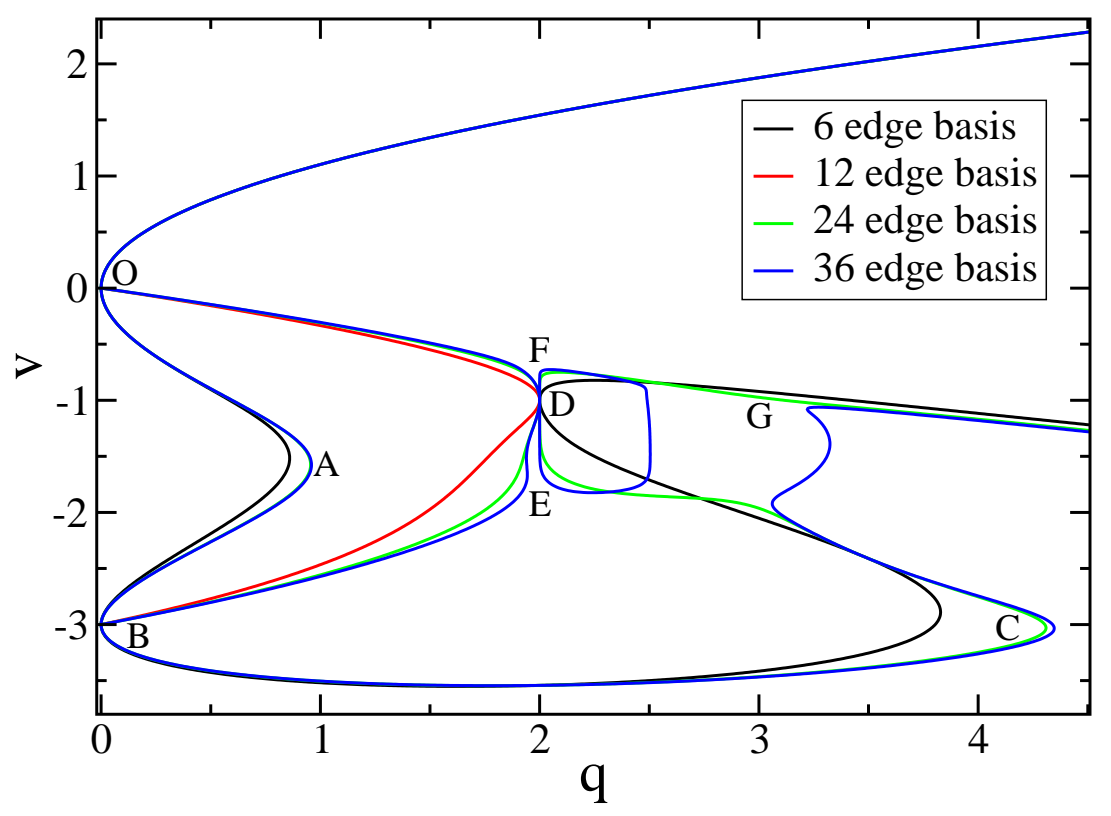

Figure 8. Manifolds $P_{B}(q, v)=0$ for various choices of bases $B$ for the kagome lattice (see text). The manifold corresponding to the basis with 6 edges is contained as a factor in the 12-edge manifold. The letters in the figure serve as a guide to the discussion in the main text.

$q=4$ we find

$$
v_{\mathrm{c}}(q=4)= \begin{cases}2.155842236 \cdots & \text { (6-edge basis) } \\ 2.156207452 \cdots & \text { (24-edge basis) } \\ 2.156223187 \cdots & \text { (36-edge basis) }\end{cases}
$$

whereas the numerics [20] gives $v_{\mathrm{c}}(q=4)=2.15620(5)$.

We defer a more detailed discussion of the ferromagnetic region to section 6, where we shall also present our own numerical results. Instead we now discuss some common features and differences of the curves and formulate some conjectures about the thermodynamical limit.

There is a branch that extends to infinite $q$ in both the ferromagnetic and antiferromagnetic $(v<0)$ regions. Both of these have the correct asymptotic behaviour $v \propto \sqrt{q}$ for all bases. Indeed, on a lattice of coordination number $z$, one needs $v \propto q^{z / 2}$ in order to ensure first-order phase coexistence between the two dominant terms (with $A=E$ and $A=\emptyset$ respectively) in the expansion (2).

With all bases, the ferromagnetic curve runs into the origin $\mathrm{O}:(q, v)=(0,0)$ with infinite slope, as is required [15] to produce a model of spanning trees. The continuation into the antiferromagnetic region bends around at $\mathrm{A}:(q, v) \approx(0.96,-1.58)$, and then goes down to the point B : $(q, v)=(0,-3)$. Since all curves pass through $(q, v)=(0,-3)$ exactly, we conjecture that this is a correct feature of the thermodynamical limit. If correct, this would imply that on the dual (diced) lattice, the problem of spanning forests 15. has a critical point with a weight per tree $w_{\mathrm{c}}=-3$. 
After bending around in $\mathrm{B}$ the curves continue in the region $v<-3$ to a point $\mathrm{C}:(q, v) \approx(4.35,-3.03)$ where they bend around again. It is interesting that $\mathrm{C}$ definitely has $q>4$, a feature that was not present in Wu's conjecture (alias the 6-edge curve).

The curves show strong finite-size effects in the domain $-3<v<0$ (excluding the branch $\mathrm{OAB}$ which has been discussed above). In particular, it might be that the point $\mathrm{C}$ will eventually move to larger $q$, or even infinity.

For all bases the curves pass through $\mathrm{D}:(q, v)=(2,-1)$ exactly, and we conjecture that this is a correct feature of the thermodynamical limit. For larger bases the branches $\mathrm{BD}$ and $\mathrm{DC}$ have a tendency to close up for $q \approx 2$ so as to form an almost vertical ray. We conjecture that in the thermodynamical limit there will be an exactly vertical ray extending between $\mathrm{E}:(q, v)=\left(2, v_{1}\right)$ and $\mathrm{F}:(q, v)=\left(2, v_{2}\right)$, for some $v_{1}<v_{2}$. *

It in then natural to assume that $\mathrm{BEC}$ and $\mathrm{OF} \infty$ will be smooth transition curves in the thermodynamical limit. The 36-edge basis shows that the space in between these two curves is likely to contain a further vertical ray. Since the properties of the Berker-Kadanoff phase can be argued to be universal [39] we conjecture that the other ray will again be located at $q=3$ [37], cf. our discussion of the square lattice above. We further conjecture that the curves $\mathrm{OAB}, \mathrm{BEC}$ and $\mathrm{OF} \infty$ will surround the Berker-Kadanoff phase. To delimit this phase to the right, one more curve is needed. The 36-edge basis provides a convincing finite-size estimate for this latter curve (starting at $(q, v) \approx(3.06,-1.92)$, bending around at $(q, v) \approx(3.32,-1.39)$ and ending at $(q, v) \approx(3.22,-1.08))$.

We believe that in the thermodynamical limit the curve $F \infty$ will pass through the point $\mathrm{G}:(q, v)=(3,-1)$ exactly. Namely, one can show [40] that the three-state zerotemperature antiferromagnet on the kagome lattice is equivalent to the corresponding four-state model on the triangular lattice. The latter is known to be critical with central charge $c=2$ (see [40] and references therein). Our finite bases locate the antiferromagnetic transition in the $q=3$ model at

$$
v_{\mathrm{c}}^{\mathrm{AF}}(q=3)= \begin{cases}-0.921400117 \cdots & \text { (6-edge basis) } \\ -0.973665377 \cdots & \text { (24-edge basis) }\end{cases}
$$

and it seems likely that this might tend to $v_{\mathrm{c}}^{\mathrm{AF}}(q=3)=-1$ in the thermodynamical limit. This is presumably the point in the phase diagram where the finite-size effects are the most important.

We should stress here that to get a reliable picture of the phase diagram it is not sufficient to study just one basis $B$, however large. Indeed it is obvious from Fig. 8 that different bases reveal different parts of the critical manifold. For instance, the 6-edge

\footnotetext{
* Note that the existence of this vertical ray is not in contradiction with the argument of Huse and Rutenberg [38] (using results of [14]) that the kagome-lattice Ising model is disordered for any $v>-1$. Indeed, the difference between sitting at $q=2$ exactly, and taking the limit $q \rightarrow 2$ in the $(q, v)$ plane phase diagram, is the quintessence of the special physics at the Beraha numbers within the antiferromagnetic region [22, 9].
} 
basis misses completely the curve OFEB. And even though the 36-edge basis gives in many respects the most precise approximation to the true critical manifold, it misses a part of the curve $\mathrm{F} \infty$ which is covered by the smaller 24-edge basis.

Quite obviously it would require more work to give numerical support for the phase diagram discussed above and determine the relevant critical properties. In section 6 we limit ourselves to a detailed investigation of the ferromagnetic transition curve and of the antiferromagnetic branch BC.

Note finally that the slope $1 / w$ with which the curve OD goes into the origin determines the critical point in a model of spanning forests [15] on the kagome lattice. The critical weight per component tree can be determined as

$$
w_{\mathrm{c}}= \begin{cases}-3.364655607 \cdots & \text { (12-edge basis) } \\ -3.553344713 \cdots & \text { (24-edge basis) } \\ -3.578781346 \cdots & \text { (36-edge basis) }\end{cases}
$$

We can also determine the critical point of the flow polynomial $\Phi(q)$, which is related to the Potts-model partition function (2) by setting $v=-q$ [23]. We find

$$
q_{\mathrm{c}}= \begin{cases}3.324717957 \cdots & \text { (6-edge basis) } \\ 3.400923464 \cdots & \text { (24-edge basis) } \\ 3.405701476 \cdots & \text { (36-edge basis) }\end{cases}
$$

By duality this can also be interpreted as the critical point of the chromatic polynomial on the diced lattice. Our results (44) agree with the crude extrapolation $q_{\mathrm{c}} \approx 3.4$ given in [49].

\section{Numerical results}

We have performed extensive numerical simulations in order to accurately locate (parts of) the critical manifold for the kagome-lattice Potts model. The objective is to assess whether the graph polynomials found in section 5 improve on Wu's conjecture (6) - and if so, by how much - when the size of the basis increases.

For a critical system, the finite-size scaling of the free energy per unit area $f(L)$ on a cylinder of circumference $L$ reads 41,42 ,

$$
f(L)=f(\infty)-\frac{\pi c}{6 L^{2}}+o\left(L^{-2}\right),
$$

where $f(\infty)$ is the free energy in the thermodynamical limit and $c$ is the central charge. This result also provides an accurate means of locating the critical point, since the numerically determined $c$ will exhibit a local extremum at criticality [43]. The precision can be improved 44 by adding a non-universal term $A / L^{4}$ to (45).

\subsection{Transfer matrix construction}

The quantity $f(L)$ can be obtained from the largest eigenvalue of the transfer matrix. There are two natural transfer directions for the kagome lattice: parallel or perpendicular 
to one third of the lattice edges. In Fig. 7 the parallel and perpendicular transfer directions are respectively horizontal and vertical. Let $\mathrm{T}_{N}$ be the transfer matrix written in a basis of states that keeps track of $N$ spins in a "time slice" - a layer perpendicular to the transfer direction. Using the basis provided by the Fortuin-Kasteleyn representation (2) then determines the number of basis states (i.e., the dimension of $\mathrm{T}_{N}$ ) as the Catalan number 45 ]

$$
C_{N}=\frac{1}{N+1}\left(\begin{array}{c}
2 N \\
N
\end{array}\right) \simeq \frac{4^{N}}{N^{3 / 2} \pi^{1 / 2}}[1+\mathcal{O}(1 / N)]
$$

The physical width $L$ is $N$ times the height (resp. $N$ times the side length) of an elementary triangle for the parallel (resp. perpendicular) transfer direction. It is therefore most efficient to choose the perpendicular direction (i.e., such that the time runs vertically in Fig. 7). Setting the side length of an elementary triangle to unity, we can henceforth identify $N=L$.

We suppose henceforth $L$ even, since otherwise the kagome lattice would not be compatible with periodic boundary conditions across the time slice. Let I, $\mathrm{J}_{i, i+1}$ and $\mathrm{D}_{i}$ be the identity, join and detach operators (see [46]) satisfying the Temperley-Lieb algebra [47] with weight $q$ per connected component. The composite operators

$$
\begin{aligned}
\mathrm{H}_{i} & =\mathrm{I}+v \mathrm{~J}_{i, i+1}, \\
\mathrm{~V}_{i} & =v \mathbf{I}+\mathrm{D}_{i}
\end{aligned}
$$

then add respectively a horizontal ("space-like") edge between the vertices $i$ and $i+1$, and a vertical ("time-like") edge a vertex $i$. The edge weight $v$ is that of (2). From these we can define an operator that builds the basic bow tie pattern of the kagome lattice:

$$
\mathrm{B}_{i}=\mathrm{H}_{i} \bigvee_{i} \mathrm{H}_{i} \mathrm{D}_{i+1} \mathrm{H}_{i} \mathrm{~V}_{i} \mathrm{H}_{i}
$$

The transfer matrix then reads (by simple inspection of Fig. (7)

$$
\mathrm{T}_{L}=\left(\prod_{i=1}^{L / 2} \mathrm{~B}_{2 i}\right)\left(\prod_{i=1}^{L / 2} \mathrm{~B}_{2 i-1}\right) \text {. }
$$

Since this propagates the time slice by four times the height of an elementary triangle, the corresponding free energy per unit area is

$$
f(L)=-\frac{1}{2 \sqrt{3} L} \log \Lambda_{\max },
$$

where $\Lambda_{\max }$ is the eigenvalue of $\mathrm{T}_{L}$ with maximal norm.

We have diagonalised $\mathrm{T}_{L}$ for $L=2,4,6, \ldots, 16$ and computed finite-size estimates $c(L)$ of the central charge $c$ from (45) with the $A / L^{4}$ term included, by using three successive sizes $(L-4, L-2, L)$. For each fixed $q=\frac{1}{10}, \frac{2}{10}, \ldots, \frac{40}{10}$ we have varied $v$ until a local extremum was found. This extremum provides an estimate of the critical coupling $\uplus$.

$\sharp$ For $q=1$ there is no finite-size dependence in $f(L)$, so the method does not work in that case. 


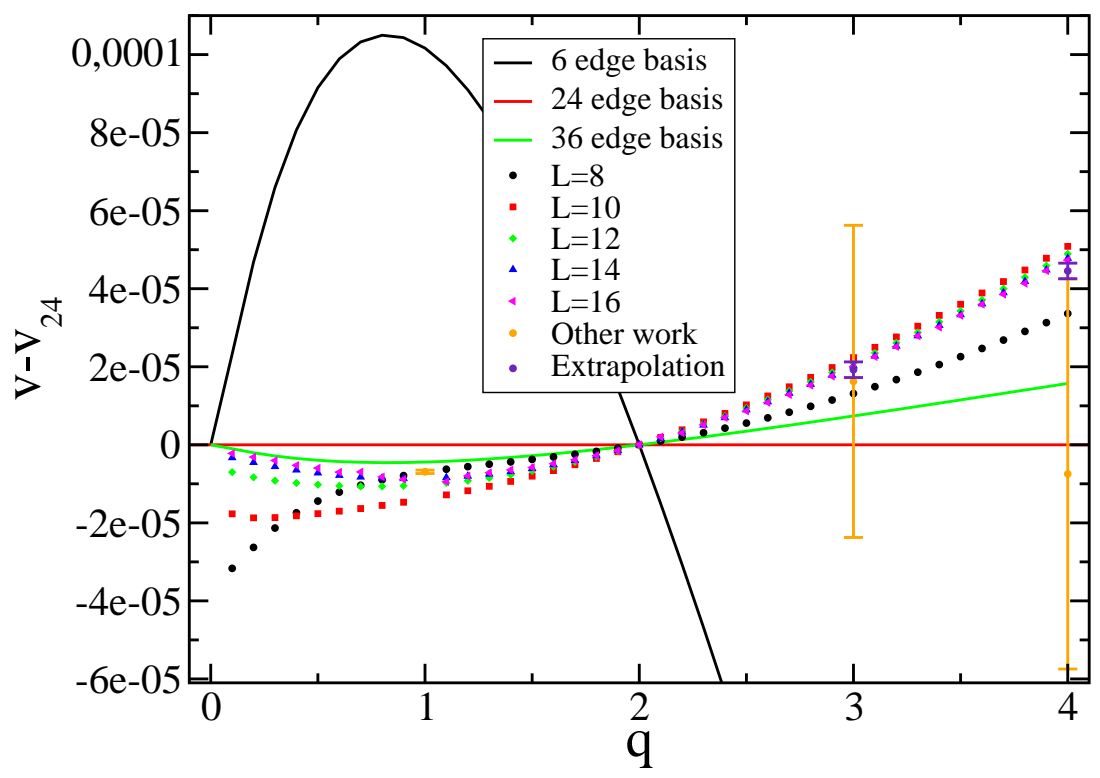

Figure 9. Estimates of the critical curve in the ferromagnetic regime, relative to the approximation provided by the 24-edge basis. The solid lines show the zeros of $P_{B}(q, v)$ for various bases $B$. The points are numerical transfer matrix results for system size $L$ (see text). Extrapolations for $L \rightarrow \infty$ are shown for $q=3$ and $q=4$. Other numerical determinations are given for $q=1, q=3$, and $q=4$ (and labelled "other work").

\subsection{Ferromagnetic critical curve}

We first present the results in the ferromagnetic region $v>0$. It is evident from Fig. 8 that the various approximations to the critical manifold are extremely close, so in order to appreciate the differences we focus on the quantity $v-v_{24}$, where $v_{24}$ is the relevant root of $P_{B}(q, v)$ using the 24-edge basis.

Fig. 9 shows our numerical results (up to size $L=16$ ) along with the approximations obtained from the graph polynomials of section 5 using bases with 6, 24 and 36 edges. For $q=3$ and $q=4$ we further show extrapolations of the numerical results:

$$
v_{\mathrm{c}}= \begin{cases}1.876459(2) & (q=3) \\ 2.156252(2) & (q=4)\end{cases}
$$

These were obtained by fitting the residual dependence in $1 / L$ to first and second-degree polynomials, and estimating the error bars by comparison and successive elimination of the data points with small $L$. We also show in the figure the previous numerical results for $q=1$ (percolation [16, 20]), $q=3$ (series expansions [18]), and $q=4$ (transfer matrix diagonalisations [20]

It is clear that at this level of precision the Wu conjecture (6) - i.e., the 6-edge basis result - is definitively invalidated. More precisely, it is ruled out by our results (51) with †† We do not show the series result [18] for $q=4$, since the corresponding error bar is several times the vertical extent of Fig. 9. The $q=3$ result of [20] is also not shown, because it is almost identical to our own extrapolation. 
a confidence level of 95 (resp. 205) standard deviations for $q=3$ (resp. $q=4$ ).

More importantly, it is obvious from Fig. 9 that the improved approximations with 24 and 36-edge bases move systematically towards the numerical results. The results from the 36-edge base are at a tiny distance from the numerics, of the order $10^{-6}$ or $10^{-7}$. It is nevertheless clear that even those results only constitute an approximation.

Note also that the numerics provides (within machine precision) the exact $v_{\mathrm{c}}$ for the Ising model $(q=2)$.

We finally remark that in a recent paper Baek et al. [48] located the critical points for $q=3$ and $q=4$ using phenomenological scaling of the internal energy crossings. These we obtained from transfer matrices (with the "parallel transfer direction" in the terminology of section 6.1). The authors of [48] claimed that these results are independent of system size, whence the results for the smallest possible systems ( $N=2$ and $N=4$ in the notation of section 6.1):

$$
\begin{array}{ll}
v_{\mathrm{c}}=1.876313463895 \cdots, & (q=3) \\
v_{\mathrm{c}}=2.156174166284 \cdots, & (q=4)
\end{array}
$$

would be exact. This claim is however not true. Internal energy crossings indeed exactly determine the critical point for the square-lattice Potts model (by an easy duality argument), and for the Ising model on more general lattices including kagome. However, for the $q \neq 2$ Potts model on the kagome lattice, the energy crossings do exhibit finitesize corrections. Note also that the results (52) are incompatible with our numerical results (51).

\subsection{Antiferromagnetic critical curve}

We have similarly investigated numerically the antiferromagnetic critical curve in the region $v<-3$ (referred to as $\mathrm{BC}$ in section 5). As in the ferromagnetic case we show the differences $v-v_{24}$ (see Fig. 10).

It is evident from the scale of the vertical axis that the finite-size effects are much larger than in the ferromagnetic region. In particular, larger bases would be needed to convincingly approximate the numerical results, especially in the region $q \simeq 4$.

One exception is again the Ising model $(q=2)$ where finite-size effects are completely absent. Indeed, all curves pass through the exact value [13] $v_{\mathrm{c}}(q=2)=$ $-\sqrt{3+2 \sqrt{3}}-1=-3.542459756 \cdots$.

Extrapolations of the finite-size numerics - obtained using the method described above-read:

$$
v_{\mathrm{c}}= \begin{cases}-3.486(2) & (q=3) \\ -3.361(14) & (q=4)\end{cases}
$$

and are shown in Fig. 10. The result for $q=3$ is in agreement with the Monte Carlo determination [49] of the critical coupling of the 3-state Potts model on the diced lattice, $v_{\mathrm{c}}^{\text {diced }}=-0.860599(4)$. The corresponding dual value $v_{\mathrm{c}}=3 / v_{\mathrm{c}}^{\text {diced }}=-3.48594(2)$ is in fact more precise than (53). 


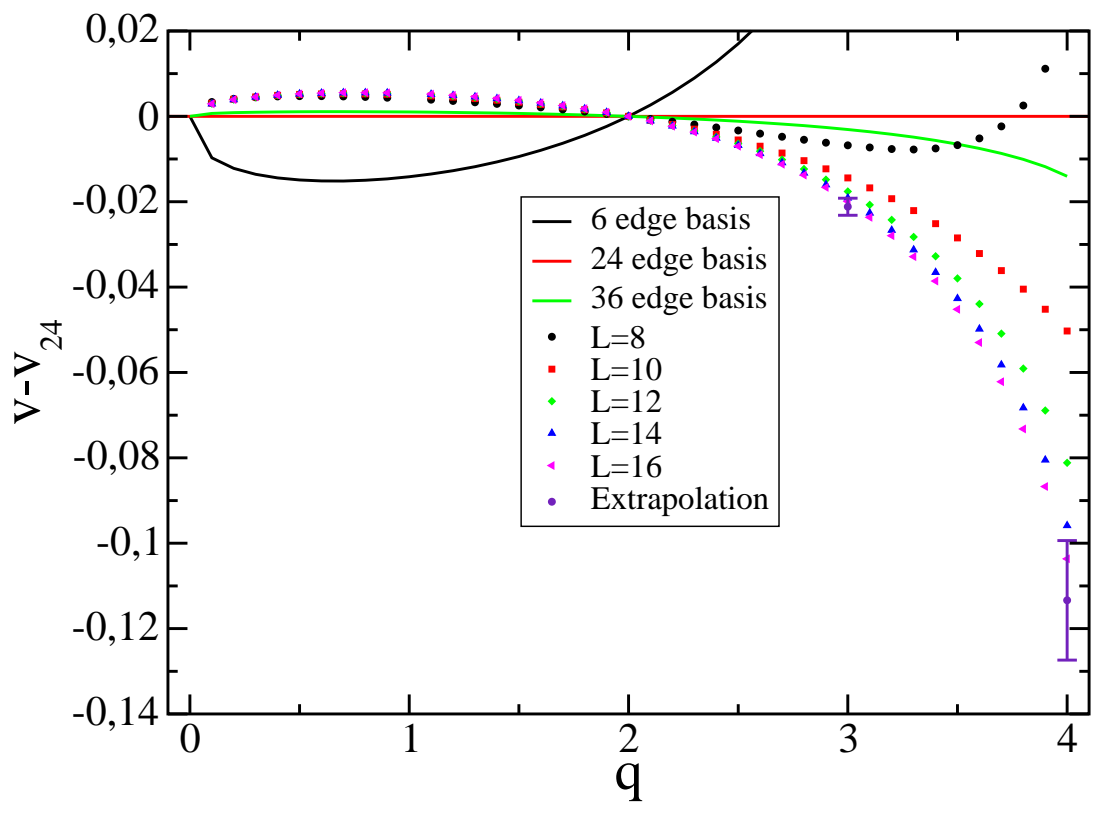

Figure 10. Estimates of the critical curve $\mathrm{BC}$ in the antiferromagnetic regime, relative to the approximation provided by the 24-edge basis. The symbols and colours have the same meaning as in Fig. 9 ,

The Monte Carlo study [49] also shows that the 4-state Potts model on the diced lattice is disordered for all $-1 \leq v^{\text {diced }}<0$. The corresponding dual statement is that for $q=4$, there is no phase transition for $-\infty<v \leq-4$. This again agrees with the fact that our critical manifolds avoid this interval.

\subsection{Critical properties}

The numerical results for the central charge along the ferromagnetic transition curve are shown in Fig. 11, They agree with the universality class of the usual ferromagnetic Potts model:

$$
c=1-\frac{6}{k(k-1)},
$$

where $q=B_{k}$ is parameterised by $k \in[2, \infty)$ through (37). In fact, the deviations from the exact result are not discernible on the scale of the figure.

The corresponding results along the antiferromagnetic curve $\mathrm{BC}$ are given in Fig. 12, Remarkably they again agree with (54). Note that there are now rather strong finite-size effects in the region $q \simeq 4$.

The result for $k=6$ - namely that the $q=3$ model at the antiferromagnetic transition (53) is in the universality class of the three-state ferromagnetic model-was previously reported in [49]. 


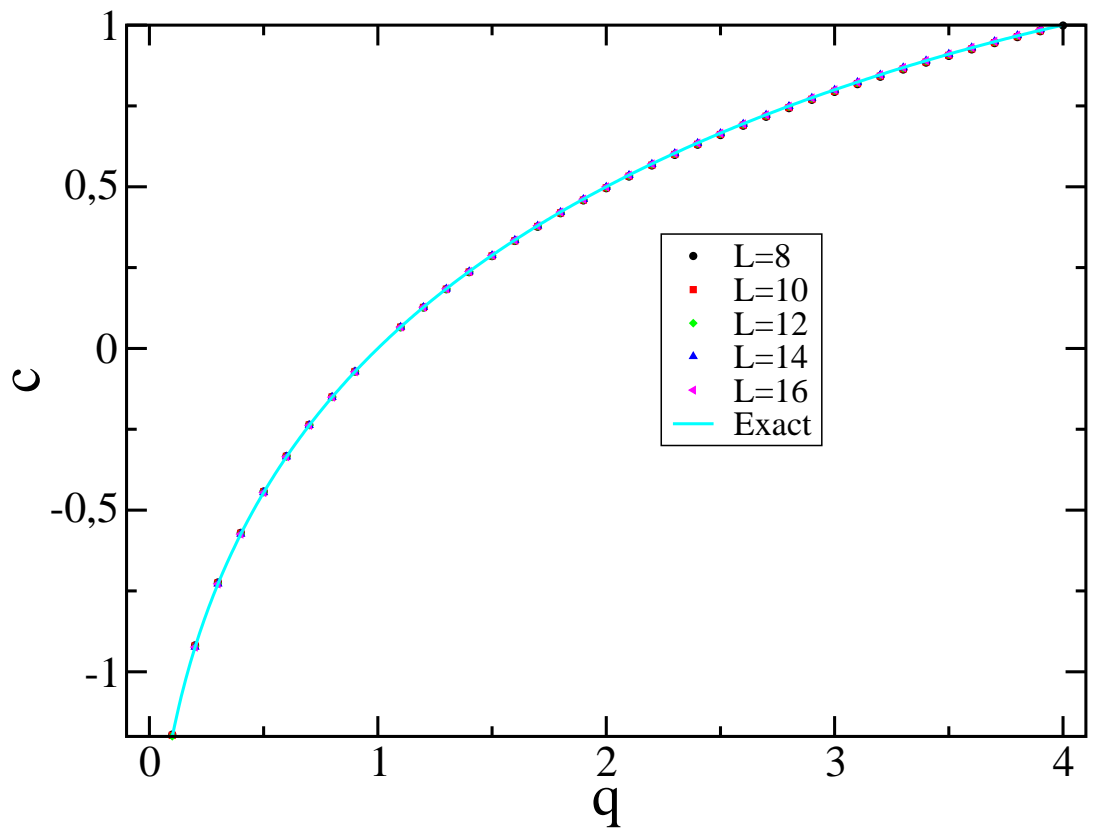

Figure 11. Central charge $c$ along the ferromagnetic transition curve.

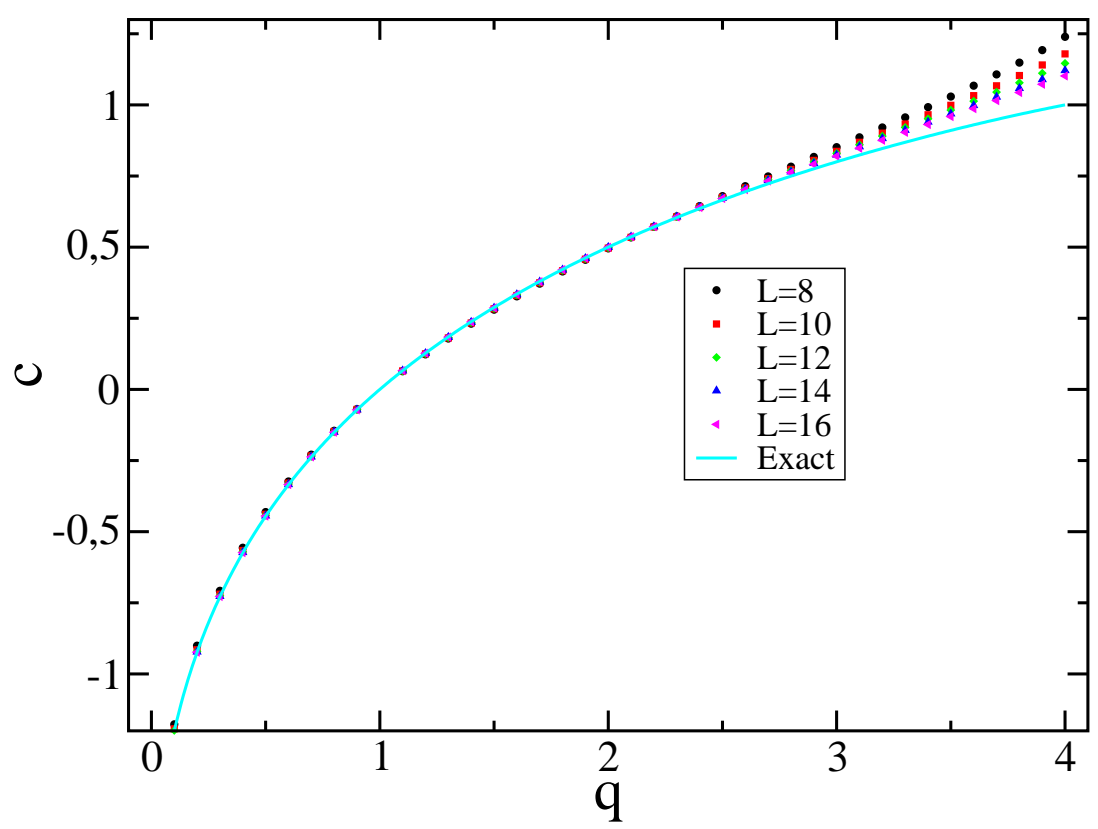

Figure 12. Central charge $c$ along the antiferromagnetic transition curve BC. 


\section{Discussion}

In this article we have defined a graph polynomial $P_{B}(q, v)$ that characterises a finite basis graph $B$ and the way it is embedded in order to tile an infinite regular lattice $G$. Being closely related to the Tutte polynomial, $P_{B}(q, v)$ can be evaluated recursively from the contraction-deletion formula (201).

The zero sets (7) were shown to reproduce well-known approximations for the critical manifolds for the Potts model on the checkerboard and kagome lattices [11] in the case of the smallest possible bases. We have shown that such approximations can be systematically ameliorated by increasing the size of the basis. In particular, the 36-edge basis for the kagome lattice provides an approximation of the critical manifold whose deviation from our high-precision numerical study is of the order $10^{-6}$ or $10^{-7}$ in the ferromagnetic regime $(v>0)$. In the antiferromagnetic regime $(v<0)$ our approximations reveal qualitatively new structures, such as singularities at the Beraha numbers (37) and the extent of the Berker-Kadanoff phase [22, 9].

The fact that the resulting 32nd-order algebraic curve still does not reproduce perfectly the numerical data leads us to conjecture that the true critical manifold for the kagome lattice is non-algebraic. It is tempting to speculate that this behaviour may be responsible for the fact that the Potts model on the kagome lattice has not yet been solved (except for the $q=2$ Ising and $q=0$ spanning tree cases).

We should add here a small caveat. We have seen that both for percolation [28] and for the general $q$-state Potts model, when they are not exact, the polynomials $P_{B}(q, v)$ provide accurate approximations for unsolved problems that improve with an increasing number of edges in $B$. This is the motivation for our conjecture that the polynomials converge to the exact critical manifold in the limit of certain infinite bases. However, as shown in [28, it is not enough that the number of edges in $B$ tends to infinity. Let us say that a generic basis is of size $N \times M$ if it contains $N$ unit cells in the vertical direction and $M$ unit cells in the horizontal direction. For example, for the kagome lattice the unit cell is the six-edge bow tie pattern shown in Fig. 3a , so that the bases of Figs. 76 and 7b are of size $2 \times 3$ and $3 \times 2$ respectively. In [28], an example was found of a lattice for which the $1 \times M$ basis prediction for increasing $M$ converged to a number that, although similar to the numerically known percolation threshold, was ruled out by simulations. Thus we should add the condition that in order to produce a sequence of algebraic curves of increasing order that converge to the exact critical manifold, both $N$ and $M$ must go to infinity simultaneously with a finite aspect ratio $\eta \equiv N / M \in(0, \infty)$. Although it is difficult to probe this at present, it seems at least plausible, if not likely, that these infinite basis predictions should also be independent of $\eta$.

An interesting feature of our findings is that in some sense the computation of the polynomials $P_{B}(q, v)$ acts as a detector of exact solvability for the underlying model. To be more precise, it seems that if the polynomial factorises for any choice of basis, shedding always the same "small" factor, the zero set of that factor provides (a part of) the exact critical manifold. We have seen this mechanism at play in the case of the square 
lattice, where the small factor is given by (3). More generally, for the square lattice with checkerboard interactions (see Fig. 2) the small factor is given by (22), leading us to conjecture that this is the exact critical manifold for the checkerboard model. We stress that exact results are only expected if the factorisation is systematic, i.e., occurs for

any $B$. As an example of the contrary, the 12-edge basis of the kagome lattice led to the fortuitous factorisation (38), and we have shown convincingly that neither of the factors provide an exact result. We should also notice that the implication does not seem to work the other way around: exact solvability does not imply factorisation. This is witnessed in particular by the case $(q, v)=(3,-1)$ of the kagome-lattice Potts model, which is exactly solvable [40], but not a zero of $P_{B}(q, v)$ for the finite bases that we have studied here.

Our work hints at several directions for future research. It would obviously be worthwhile having a more efficient means of dealing with yet larger bases. This would require finding an alternative definition of $P_{B}(q, v)$ that does not refer to contractiondeletion. The study of the critical manifolds for other lattices is another possibility. Finally, it is quite possible that $P_{B}(q, v)$ conceals some graph theoretical applications.

\section{Acknowledgements}

The work of JLJ was supported by the Agence Nationale de la Recherche (grant ANR-10BLAN-0414: DIME) and the Institut Universitaire de France. This work was partially (CRS) performed under the auspices of the U.S. Department of Energy by Lawrence Livermore National Laboratory under Contract DE-AC52-07NA27344. JLJ wishes to express his gratitude to Jesús Salas for collaboration on a related project [21]. Both authors thank J. Salas and F.Y. Wu for some interesting comments on the manuscript. We are also grateful to the Mathematical Sciences Research Institute at the University of California, Berkeley for hospitality during the programme on Random Spatial Processes where this work was initiated.

[1] R.B. Potts, Proc. Camb. Phil. Soc. 48, 106-109 (1952).

[2] F.Y. Wu, Rev. Mod. Phys. 54, 235 (1982).

[3] R.J. Baxter, Exactly solved models in statistical mechanics, (Academic Press, London, 1982).

[4] C.M. Fortuin and P.W. Kasteleyn, Physica 57, 536-564 (1972).

[5] L. Onsager, Phys. Rev. 65, 117-149 (1944).

[6] R.J. Baxter, J. Phys. C 6, L445-L448 (1973).

[7] R.J. Baxter, H.N.V. Temperley and S.E. Ashley, Proc. R. Soc. Lond. A 358, 535-559 (1978).

[8] F.Y. Wu, Phys. Rev. E 81, 061110 (2010).

[9] J.L. Jacobsen and H. Saleur, Nucl. Phys. B 743, 207-248 (2006).

[10] R.J. Baxter, Proc. Roy. Soc. London Ser. A 383, 43 (1982).

[11] F.Y. Wu, J. Phys. C 12, 645 (1979).

[12] C. Tsallis, J. Phys. C 15, L757 (1982).

[13] K. Kano and S. Naya, Prog. Theor. Phys. 10, 158 (1953).

[14] J.H. Barry, M. Khatun and T. Tanaka, Phys. Rev. B 37, 5193 (1988).

[15] J.L. Jacobsen, J. Salas and A.D. Sokal, J. Stat. Phys. 119, 1153-1281 (2005).

[16] R.M. Ziff and H. Gu, Phys. Rev. E 79, 020102(R) (2009).

[17] R.M. Ziff and P.N. Suding, J. Phys. A: Math. Gen. 30, 5351 (1997). 
[18] I. Jensen, A.J. Guttmann and I.G. Enting, J. Phys. A: Math. Gen. 30, 8067 (1997).

[19] J.L. Monroe, Phys. Rev. E 67, 017103 (2003).

[20] C. Ding, Z. Fu, W. Guo and F.Y. Wu, Phys. Rev. E 81, 061111 (2010).

[21] J.L. Jacobsen and J. Salas, unpublished (2008).

[22] H. Saleur, Nucl. Phys. B 360, 219 (1991).

[23] A.D. Sokal, The multivariate Tutte polynomial (alias Potts model) for graphs and matroids, in B.S.

Webb (ed.) Surveys in combinatorics, Lond. Math. Soc. Lect. Note Ser. 327, 173 (2005).

[24] C.R. Scullard, arXiv:1111.1061.

[25] C.R. Scullard and R.M. Ziff, Phys. Rev. Lett. 100, 185701 (2008).

[26] C.R. Scullard and R.M. Ziff, J. Stat. Mech. 2010, P03021.

[27] C.R. Scullard, J. Stat. Mech. 2011, P09022.

[28] C.R. Scullard, In preparation.

[29] F.Y. Wu and K.Y. Lin, J. Phys. A 13, 629-636 (1980).

[30] I.G. Enting, J. Phys. A: Math. Gen. 20, L917 (1987).

[31] F.Y. Wu, private communication.

[32] J.-M. Maillard, Chinese J. Phys. 40, 327-378 (2002); arXiv: cond-mat/0205063v2.

[33] C.R. Scullard, Phys. Rev. E 73, 016107 (2006).

[34] R.M. Ziff, Phys. Rev. E 73, 016134 (2006).

[35] F.Y. Wu, Phys. Rev. Lett. 96, 090602 (2006).

[36] J.L. Jacobsen and J. Salas, J. Stat. Phys. 122, 705-760 (2006).

[37] J.L. Jacobsen and J. Salas, Nucl. Phys. B 783, 238-296 (2007).

[38] D.A. Huse and A.D. Rutenberg, Phys. Rev. B 45, 7536-7539 (1992).

[39] J.L. Jacobsen and H. Saleur, J. Stat. Phys. 132, 707-719 (2008).

[40] C.M. and M.E.J. Newman, J. Stat. Phys. 99, 629-660 (2000).

[41] H.W.J. Blöte, J.L. Cardy and M.P. Nightingale, Phys. Rev. Lett. 56, 742 (1986).

[42] I. Affleck, Phys. Rev. Lett. 56, 746 (1986).

[43] A.B. Zamolodchikov, Sov. Phys. JETP Lett. 43, 730 (1986) [Zh. Eksp. Teor. Fiz. 43, 565 (1986)].

[44] J.L. Jacobsen and J.L. Cardy, Nucl. Phys. B 515, 701-742 (1998).

[45] H.W.J. Blöte and M.P. Nightingale, Physica A 112, 405-465 (1982).

[46] J. Salas and A.D. Sokal, J. Stat. Phys. 104, 609-699 (2001).

[47] H.N.V. Temperley and E.H. Lieb, Proc. R. Soc. Lond. A 322, 251-280 (1971).

[48] S.K. Baek, H. Mäkelä, P. Minnhagen and B.J. Kim, Phys. Rev. E 83, 061104 (2011).

[49] R. Kotecký, J. Salas and A.D. Sokal, Phys. Rev. Lett. 101, 030601 (2008).

\section{Appendix}

Here, we report the graph polynomials $P_{B}(q, v)$ for the various bases $B$ and graphs $G$ considered.

Square lattice

The 16-edge basis of Fig. $5 \mathrm{~b}$ :

$$
\begin{aligned}
& \left(q-v^{2}\right)\left(q+4 v+v^{2}\right)\left(q^{6}+12 q^{5} v+68 q^{4} v^{2}+232 q^{3} v^{3}+4 q^{4} v^{3}\right. \\
+ & 516 q^{2} v^{4}+40 q^{3} v^{4}+q^{4} v^{4}+736 q v^{5}+192 q^{2} v^{5}+8 q^{3} v^{5}+576 v^{6} \\
+ & 504 q v^{6}+52 q^{2} v^{6}+736 v^{7}+192 q v^{7}+8 q^{2} v^{7}+516 v^{8}+40 q v^{8} \\
+ & \left.q^{2} v^{8}+232 v^{9}+4 q v^{9}+68 v^{10}+12 v^{11}+v^{12}\right)
\end{aligned}
$$

The 32-edge basis of Fig. 56:

$$
\left(q-v^{2}\right)\left(q+4 v+v^{2}\right)\left(q^{14}+28 q^{13} v+384 q^{12} v^{2}+3424 q^{11} v^{3}\right.
$$


Critical manifold of the kagome-lattice Potts model

$$
\begin{aligned}
& +4 q^{12} v^{3}+22240 q^{10} v^{4}+112 q^{11} v^{4}+q^{12} v^{4}+111744 q^{9} v^{5} \\
& +1536 q^{10} v^{5}+24 q^{11} v^{5}+450016 q^{8} v^{6}+13632 q^{9} v^{6}+304 q^{10} v^{6} \\
& +1484032 q^{7} v^{7}+87520 q^{8} v^{7}+2688 q^{9} v^{7}+8 q^{10} v^{7}+4053840 q^{6} v^{8} \\
& +430560 q^{7} v^{8}+18520 q^{8} v^{8}+176 q^{9} v^{8}+q^{10} v^{8}+9198080 q^{5} v^{9} \\
& +1676320 q^{6} v^{9}+104032 q^{7} v^{9}+2048 q^{8} v^{9}+20 q^{9} v^{9}+17211584 q^{4} v^{10} \\
& +5249504 q^{5} v^{10}+484160 q^{6} v^{10}+16288 q^{7} v^{10}+256 q^{8} v^{10} \\
& +25996800 q^{3} v^{11}+13255488 q^{4} v^{11}+1867840 q^{5} v^{11}+98816 q^{6} v^{11} \\
& +2368 q^{7} v^{11}+12 q^{8} v^{11}+30259968 q^{2} v^{12}+26606400 q^{3} v^{12} \\
& +5923440 q^{4} v^{12}+479248 q^{5} v^{12}+17256 q^{6} v^{12}+208 q^{7} v^{12}+q^{8} v^{12} \\
& +24551424 q v^{13}+40761344 q^{2} v^{13}+15114304 q^{3} v^{13}+1896032 q^{4} v^{13} \\
& +100736 q^{5} v^{13}+2240 q^{6} v^{13}+16 q^{7} v^{13}+10616832 v^{14}+43298304 q v^{14} \\
& +29706752 q^{2} v^{14}+6060928 q^{3} v^{14}+484352 q^{4} v^{14}+16800 q^{5} v^{14} \\
& +240 q^{6} v^{14}+24551424 v^{15}+40761344 q v^{15}+15114304 q^{2} v^{15} \\
& +1896032 q^{3} v^{15}+100736 q^{4} v^{15}+2240 q^{5} v^{15}+16 q^{6} v^{15}+30259968 v^{16} \\
& +26606400 q v^{16}+5923440 q^{2} v^{16}+479248 q^{3} v^{16}+17256 q^{4} v^{16} \\
& +208 q^{5} v^{16}+q^{6} v^{16}+25996800 v^{17}+13255488 q v^{17}+1867840 q^{2} v^{17} \\
& +98816 q^{3} v^{17}+2368 q^{4} v^{17}+12 q^{5} v^{17}+17211584 v^{18}+5249504 q v^{18} \\
& +484160 q^{2} v^{18}+16288 q^{3} v^{18}+256 q^{4} v^{18}+9198080 v^{19}+1676320 q v^{19} \\
& +104032 q^{2} v^{19}+2048 q^{3} v^{19}+20 q^{4} v^{19}+4053840 v^{20}+430560 q v^{20} \\
& +18520 q^{2} v^{20}+176 q^{3} v^{20}+q^{4} v^{20}+1484032 v^{21}+87520 q v^{21} \\
& +2688 q^{2} v^{21}+8 q^{3} v^{21}+450016 v^{22}+13632 q v^{22}+304 q^{2} v^{22} \\
& +111744 v^{23}+1536 q v^{23}+24 q^{2} v^{23}+22240 v^{24}+112 q v^{24}+q^{2} v^{24} \\
& \left.+3424 v^{25}+4 q v^{25}+384 v^{26}+28 v^{27}+v^{28}\right)
\end{aligned}
$$

Kagome lattice

The 24-edge basis of Fig. 7b:

$$
\begin{aligned}
& q^{12}+24 q^{11} v+276 q^{10} v^{2}+2016 q^{9} v^{3}+8 q^{10} v^{3}+10452 q^{8} v^{4} \\
+ & 168 q^{9} v^{4}+40680 q^{7} v^{5}+1680 q^{8} v^{5}+122384 q^{6} v^{6}+10564 q^{7} v^{6} \\
+ & 28 q^{8} v^{6}+287760 q^{5} v^{7}+46440 q^{6} v^{7}+504 q^{7} v^{7}+525096 q^{4} v^{8} \\
+ & 149724 q^{5} v^{8}+4272 q^{6} v^{8}+718704 q^{3} v^{9}+358456 q^{4} v^{9}+22376 q^{5} v^{9} \\
+ & 56 q^{6} v^{9}+673920 q^{2} v^{10}+618792 q^{3} v^{10}+78864 q^{4} v^{10}+864 q^{5} v^{10} \\
+ & 331776 q v^{11}+682128 q^{2} v^{11}+183864 q^{3} v^{11}+5976 q^{4} v^{11} \\
+ & 270432 q v^{12}+225684 q^{2} v^{12}+20700 q^{3} v^{12}+96 q^{4} v^{12}-248832 v^{13} \\
- & 95256 q v^{13}+10320 q^{2} v^{13}+264 q^{3} v^{13}-613008 v^{14}-224916 q v^{14} \\
- & 11592 q^{2} v^{14}-108 q^{3} v^{14}-723600 v^{15}-143536 q v^{15}-3416 q^{2} v^{15} \\
- & 8 q^{3} v^{15}-550377 v^{16}-53844 q v^{16}-444 q^{2} v^{16}-303288 v^{17} \\
- & 13512 q v^{17}-24 q^{2} v^{17}-127636 v^{18}-2284 q v^{18}-41784 v^{19} \\
- & 240 q v^{19}-10590 v^{20}-12 q v^{20}-2024 v^{21}-276 v^{22}-24 v^{23}-v^{24}
\end{aligned}
$$

The 36-edge basis of Fig. 7b:

$$
q^{18}+36 q^{17} v+630 q^{16} v^{2}+7128 q^{15} v^{3}+12 q^{16} v^{3}+58503 q^{14} v^{4}
$$




$$
\begin{aligned}
& +396 q^{15} v^{4}+370440 q^{13} v^{5}+6336 q^{14} v^{5}+1878670 q^{12} v^{6}+65322 q^{13} v^{6} \\
& +66 q^{14} v^{6}+7817940 q^{11} v^{7}+486456 q^{12} v^{7}+1980 q^{13} v^{7} \\
& +27122841 q^{10} v^{8}+2780142 q^{11} v^{8}+28710 q^{12} v^{8}+79228584 q^{9} v^{9} \\
& +12642612 q^{10} v^{9}+267216 q^{11} v^{9}+220 q^{1} 2 v^{9}+195849552 q^{8} v^{10} \\
& +46787952 q^{9} v^{10}+1787790 q^{10} v^{10}+6000 q^{11} v^{10}+409851792 q^{7} v^{11} \\
& +142829784 q^{8} v^{11}+9120348 q^{9} v^{11}+78876 q^{10} v^{11}+722497068 q^{6} v^{12} \\
& +361945104 q^{7} v^{12}+36695373 q^{8} v^{12}+662258 q^{9} v^{12}+585 q^{10} v^{12} \\
& +1059589512 q^{5} v^{13}+760782804 q^{6} v^{13}+118640916 q^{7} v^{13} \\
& +3964788 q^{8} v^{13}+14424 q^{9} v^{13}+1261019664 q^{4} v^{14}+1313088660 q^{5} v^{14} \\
& +310356054 q^{6} v^{14}+17868048 q^{7} v^{14}+169878 q^{8} v^{14}+54 q^{9} v^{14} \\
& +1159847424 q^{3} v^{15}+1813868856 q^{4} v^{15}+652644588 q^{5} v^{15} \\
& +62170040 q^{6} v^{15}+1258416 q^{7} v^{15}+2472 q^{8} v^{15}+743510016 q^{2} v^{16} \\
& +1892524176 q^{3} v^{16}+1072172331 q^{4} v^{16}+167153268 q^{5} v^{16} \\
& +6467142 q^{6} v^{16}+39798 q^{7} v^{16}+12 q^{8} v^{16}+251942400 q v^{17} \\
& +1290209472 q^{2} v^{17}+1270157004 q^{3} v^{17}+334649052 q^{4} v^{17} \\
& +23635824 q^{5} v^{17}+347148 q^{6} v^{17}+528 q^{7} v^{17}+315207936 q v^{18} \\
& +827146404 q^{2} v^{18}+431966778 q^{3} v^{18}+57655440 q^{4} v^{18} \\
& +1800882 q^{5} v^{18}+8596 q^{6} v^{18}-188956800 v^{19}-193284144 q v^{19} \\
& +116524980 q^{2} v^{19}+59677656 q^{3} v^{19}+4411884 q^{4} v^{19}+51288 q^{5} v^{19} \\
& +12 q^{6} v^{19}-678249936 v^{20}-773787816 q v^{20}-180556083 q^{2} v^{20} \\
& -11670198 q^{3} v^{20}-299721 q^{4} v^{20}-3066 q^{5} v^{20}-1195116768 v^{21} \\
& - \\
& + \\
& +
\end{aligned}
$$

The 36-edge basis of Fig. 77d:

$$
\begin{aligned}
& q^{18}+36 q^{17} v+630 q^{16} v^{2}+7128 q^{15} v^{3}+12 q^{16} v^{3}+58506 q^{14} v^{4} \\
+ & 396 q^{15} v^{4}+370548 q^{13} v^{5}+6336 q^{14} v^{5}+1880512 q^{12} v^{6}+65334 q^{13} v^{6} \\
+ & 66 q^{14} v^{6}+7837704 q^{11} v^{7}+486876 q^{12} v^{7}+1980 q^{13} v^{7} \\
+ & 27272076 q^{10} v^{8}+2787144 q^{11} v^{8}+28728 q^{12} v^{8}+80068176 q^{9} v^{9} \\
+ & 12716088 q^{10} v^{9}+267888 q^{11} v^{9}+220 q^{12} v^{9}+199480572 q^{8} v^{10} \\
+ & 47328210 q^{9} v^{10}+1799508 q^{10} v^{10}+6018 q^{11} v^{10}+422114976 q^{7} v^{11} \\
+ & 145763088 q^{8} v^{11}+9245904 q^{9} v^{11}+79656 q^{10} v^{11}+754976880 q^{6} v^{12} \\
+ & 374020200 q^{7} v^{12}+37614312 q^{8} v^{12}+676604 q^{9} v^{12}+612 q^{10} v^{12}
\end{aligned}
$$




$$
\begin{aligned}
& +1126577808 q^{5} v^{13}+798846408 q^{6} v^{13}+123476376 q^{7} v^{13} \\
& +4118160 q^{8} v^{13}+15432 q^{9} v^{13}+1366323552 q^{4} v^{14}+1404553176 q^{5} v^{14} \\
& +329074176 q^{6} v^{14}+18944598 q^{7} v^{14}+186240 q^{8} v^{14}+90 q^{9} v^{14} \\
& +1280396160 q^{3} v^{15}+1977683904 q^{4} v^{15}+706012560 q^{5} v^{15} \\
& +67401848 q^{6} v^{15}+1413336 q^{7} v^{15}+3528 q^{8} v^{15}+834582528 q^{2} v^{16} \\
& +2099656080 q^{3} v^{16}+1181501136 q^{4} v^{16}+184956942 q^{5} v^{16} \\
& +7412292 q^{6} v^{16}+53976 q^{7} v^{16}+36 q^{8} v^{16}+286654464 q v^{17} \\
& +1451444832 q^{2} v^{17}+1419423912 q^{3} v^{17}+375629268 q^{4} v^{17} \\
& +27409380 q^{5} v^{17}+457176 q^{6} v^{17}+1152 q^{7} v^{17}+358691328 q v^{18} \\
& +931971420 q^{2} v^{18}+487209582 q^{3} v^{18}+66732144 q^{4} v^{18} \\
& +2296374 q^{5} v^{18}+15436 q^{6} v^{18}+6 q^{7} v^{18}-214990848 v^{19} \\
& -217611360 q v^{19}+128909448 q^{2} v^{19}+67192668 q^{3} v^{19} \\
& +5321868 q^{4} v^{19}+80928 q^{5} v^{19}+144 q^{6} v^{19}-765345024 v^{20} \\
& -871306632 q v^{20}-208435194 q^{2} v^{20}-14587674 q^{3} v^{20} \\
& -406632 q^{4} v^{20}-4050 q^{5} v^{20}-1334035008 v^{21}-1023060564 q v^{21} \\
& -184223328 q^{2} v^{21}-10352660 q^{3} v^{21}-178764 q^{4} v^{21}-540 q^{5} v^{21} \\
& -1528264908 v^{22}-755329716 q v^{22}-87028104 q^{2} v^{22}-2969586 q^{3} v^{22} \\
& -25248 q^{4} v^{22}-18 q^{5} v^{22}-1299190104 v^{23}-409417512 q v^{23} \\
& -28747908 q^{2} v^{23}-554952 q^{3} v^{23}-1956 q^{4} v^{23}-873980685 v^{24} \\
& -173604762 q v^{24}-7119900 q^{2} v^{24}-72934 q^{3} v^{24}-72 q^{4} v^{24} \\
& -482643540 v^{25}-59258256 q v^{25}-1341372 q^{2} v^{25}-6684 q^{3} v^{25} \\
& -223371438 v^{26}-16435896 q v^{26}-188820 q^{2} v^{26}-396 q^{3} v^{26} \\
& -87528036 v^{27}-3687672 q v^{27}-18852 q^{2} v^{27}-12 q^{3} v^{27} \\
& -29116983 v^{28}-657756 q v^{28}-1194 q^{2} v^{28}-8194248 v^{29} \\
& -90204 q v^{29}-36 q^{2} v^{29}-1932752 v^{30}-8964 q v^{30}-376032 v^{31} \\
& -576 q v^{31}-58875 v^{32}-18 q v^{32}-7140 v^{33}-630 v^{34}-36 v^{35}-v^{36}
\end{aligned}
$$

